\title{
Second spectrum of Chromium (Cr II), Part II: Radiative lifetimes and oscillator strengths of transitions depopulating low lying $3 \mathrm{~d}^{4} 4 \mathrm{p}$ levels
}

\author{
Safa Bouazza ${ }^{\mathrm{a}, *}$, Pascal Quinet ${ }^{\mathrm{b}, \mathrm{c}}$, Patrick Palmeri ${ }^{\mathrm{b}}$ \\ a Département de Physique, Université de Reims-Champagne, UFR SEN, BP 1039 F-51687 Reims Cedex2, France \\ ${ }^{\mathrm{b}}$ Physique Atomique et Astrophysique, Université de Mons-UMONS, B-7000 Mons, Belgium \\ ' IPNAS, Université de Liège, B-4000 Liège, Belgium
}

\section{A R T I C L E I N F O}

\section{Article history:}

Received 2 May 2017

Received in revised form 22 May 2017

Accepted 28 May 2017

Available online 20 June 2017

\begin{abstract}
A B S T R A C T
Radiative lifetime and oscillator strength values were computed by means of a pseudo-relativistic Hartree-Fock model including core-polarization and compared successfully with experimental data given in the literature for respectively numerous low-lying $\mathrm{Cr}$ II $3 \mathrm{~d}^{4} 4 \mathrm{p}$ levels, up to $54784 \mathrm{~cm}^{-1}$, and transitions depopulating these levels. Then transition probability and branching fraction data were also deduced in the wavelength range 1825-94400 Å. Furthermore the extracted radial integral values, obtained with the help of the oscillator strength parameterization method, are given for involved transitions in this work, i.e. $3 d^{4} 4 s-3 d^{4} 4 p, 3 d^{5}-3 d^{4} 4 p$ and $3 d^{4} 4 p-3 d^{4} 4 d$. We confirm our observed previous general trends, noting once again a decreasing of transition radial integral values with filling $n d$ shells of the same principal quantum numbers for $n \mathrm{~d}^{k}(n+1) \mathrm{s} \rightarrow n \mathrm{~d}^{k}(n+1)$ p transitions.
\end{abstract}

(c) 2017 Elsevier Inc. All rights reserved.

\footnotetext{
* Corresponding author.

E-mail address: safa.bouazza@univ-reims.fr (S. Bouazza).
} 
Contents

1. Introduction...1m.1.

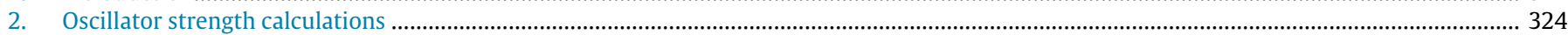

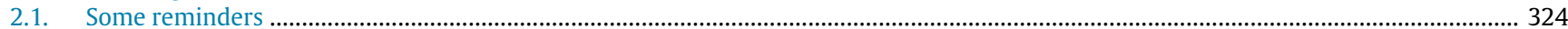

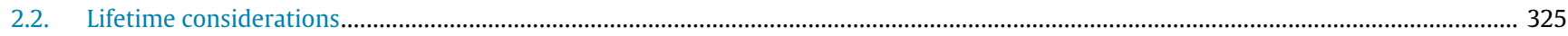

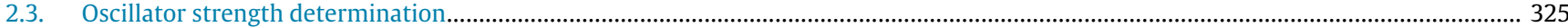

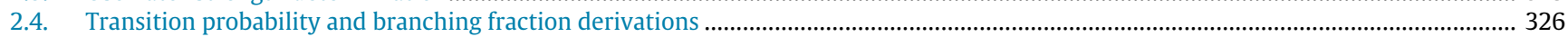

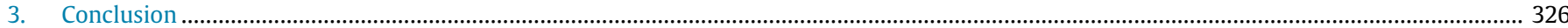

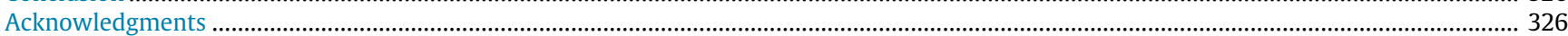

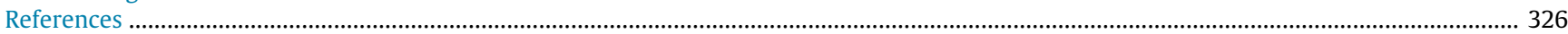

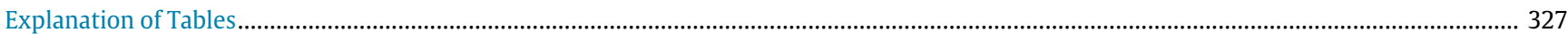

Table 1. Comparison between HFR+CPOL radiative lifetimes (in ns) and available experimental data for low-lying $3 \mathrm{~d}^{4} 4 \mathrm{p}$ energy levels

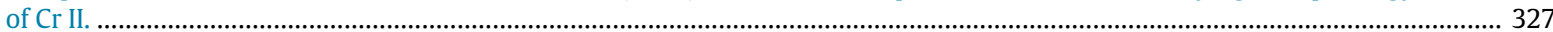

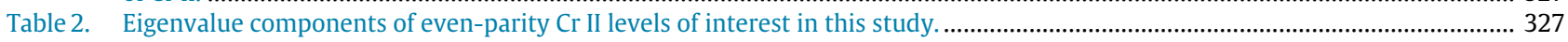

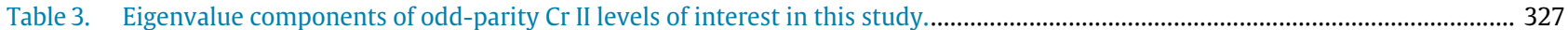

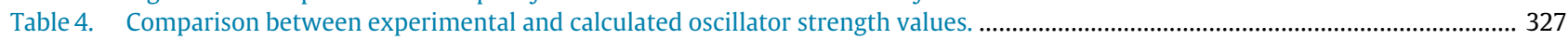

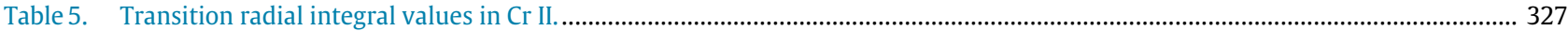

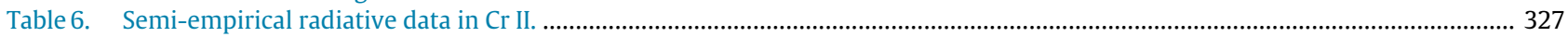

\section{Introduction}

The importance of accurate wavelengths and level energy values is now highly recognized in atomic physics when studying hyperfine structure, isotope shifts, oscillator strengths or transition probabilities of (sometimes blended) stellar spectral lines. It is particularly difficult to disentangle the blends, in the case of the elements of the $3 \mathrm{~d}$ group due to their high relative abundance and line-rich spectra. Fortunately now we have the chance to go through high resolution spectra recorded with Fourier Transform spectrometers (FTS) which have the potential to improve significantly the accuracy of the first-given wavelengths and then the precision of energy levels by at least an order of magnitude. For $\mathrm{Cr}$ II the most recent complete compilation of energy level data is that of Sugar \& Corliss [1], based on the analysis of Kiess [2] who reported for the first time observations of spectra excited in direct current arcs and condensed sparks between chromium electrodes. The latter author succeeded in classifying 1843 lines linking 138 even-parity levels of $3 d^{5}, 3 d^{4} 4 s$ and $3 d^{3} 4 s^{2}$ configurations with $1393 d^{4} 4$ p odd-parity levels. Johansson et al. [3] extended the Kiess study a half century later, particularly in the near-infrared region and analyzed 450 additional levels. Sansonetti et al. [4-6] reported in turn new observations of $\mathrm{Cr}$ II some years later, in the nearultraviolet region $1140-3400 \AA$, and also up to the infrared region: 2850-37900 Å, using a $10.7 \mathrm{~m}$ normal incidence spectrograph and an FT700 vacuum ultraviolet Fourier transform spectrometer. These new measurements have been used to revise most of the $\mathrm{Cr}$ II known levels [5-8]. These reanalyzes have permitted to exchange assignments of some levels classified in earlier lists of energy levels, to shift positions of some quartets like $3 d^{4} 5 d^{4} F_{J}$ for instance and to predict some still missing energy levels. In our previous works, when determining radiative parameter values of some ions we have observed as general trends a decreasing of transition radial integral values with filling nd-shells of the same principal quantum numbers for $n \mathrm{~d}^{k}(n+1) \mathrm{s} \rightarrow n \mathrm{~d}^{k}(n+1) \mathrm{p}$ transitions. In the present work one of our aims is to extend the study of transition radial integral behavior to another ion, located in the same row of the periodic Table, namely $\mathrm{Cr}$ II, and by the way to check our predicted transition radial integral value given in [9]. To extract this latter value we can take advantage of experimental oscillator strengths or transition probabilities, plentifully available in literature [10-14].

\section{Oscillator strength calculations}

\subsection{Some reminders}

We open this paragraph by reminding the reader of important relations we have made use in this work, split in several parts dealing each one with particular transitions: $3 d^{4} 4 s-3 d^{4} 4 p$, $3 d^{4} 4 p-3 d^{4} 5 s, 3 d^{4} 4 p-3 d^{4} 4 d, \ldots$ The oscillator strength $f\left(\gamma \gamma^{\prime}\right)$ for the transition between two levels $|\gamma J\rangle$ and $\left|\gamma^{\prime} J^{\prime}\right\rangle$ of an atom or molecule with statistical weights $g=(2 J+1)$ and $g^{\prime}=\left(2 J^{\prime}+1\right)$ respectively, is a dimensionless physical quantity, expressing the probability of absorption or emission in this transition between these two levels and related to the transition probability $W\left(\gamma \gamma^{\prime}\right)$ by work [15]

$W\left(\gamma \gamma^{\prime}\right)=\frac{2 \omega^{2} e^{2}}{m c^{2}} f\left(\gamma \gamma^{\prime}\right)$

where $m$ and $e$ are the electron mass and charge, $c$ is the velocity of light, $\gamma$ describes the initial quantum state, $\omega=\frac{E(\gamma)-E\left(\gamma^{\prime}\right)}{\hbar}, E(\gamma)$ is the energy of the initial state. The quantities with primes refer to the final state. For the electric dipole transitions, the weighted oscillator strength $g f$ is related to the line strength $S$ [15]

$g f=8 \pi^{2} m c a_{0}^{2} \frac{\sigma}{3 h} S=303.76 \times 10^{-8} \sigma S$

where $a_{0}$ is the Bohr radius, $\sigma=\frac{E(\gamma)-E\left(\gamma^{\prime}\right)}{h c}$ and $h$ is Planck's constant. The electric dipole line strength is defined by

$S=\left|\left\langle\gamma J\left\|P^{1}\right\| \gamma^{\prime} J^{\prime}\right\rangle\right|^{2}$

This quantity is a measure of the total strength of the spectral line, including all possible transitions. The tensorial operator $P^{1}$ (first order) given in units of $e a_{0}$ in the reduced matrix element stands for the electric dipole moment. To obtain the $g f$ value, we need to calculate initially $S$, or preferably its square root. For multiconfiguration systems, the wavefunctions $|\gamma J\rangle$ and $\left|\gamma^{\prime} J^{\prime}\right\rangle$ are expanded in terms of a set of basis functions $|\phi S L J\rangle$ and $\left|\phi^{\prime} S^{\prime} L^{\prime} J^{\prime}\right\rangle$, respectively

$$
\begin{gathered}
|\gamma J\rangle=\sum_{i} c_{i}\left|\phi_{i} S_{i} L_{i} J\right\rangle \\
\left|\gamma^{\prime} J^{\prime}\right\rangle=\sum_{j} c_{j}^{\prime}\left|\phi_{j}^{\prime} S_{j}^{\prime} L_{j}^{\prime} J^{\prime}\right\rangle .
\end{gathered}
$$


The square root of the line strength may be written in the following form

$S_{\gamma \gamma^{\prime}}^{1 / 2}=\sum_{i} \sum_{j} c_{i} c_{j}\left\langle\phi_{i} S_{i} L_{i} J\left\|P^{1}\right\| \phi_{j}^{\prime} S_{j}^{\prime} L_{j}^{\prime} J^{\prime}\right\rangle$.

In this work we have recurred to the eigenvector amplitudes obtained by a parametric analysis of multiconfiguration systems of $\mathrm{Cr}$ II reported in our previous paper [8].

The even system contains 11 configurations: $3 d^{5}+3 d^{4} n s(n=$ $4-9)+3 d^{4} n^{\prime} d\left(n^{\prime}=4-6\right)+3 d^{3} 4 s^{2}$, and the odd system contains 6 configurations: $3 d^{4} 4 p+3 d^{4} 5 p+3 d^{4} 6 p+3 d^{3} 4 s 4 p+3 d^{4} 4 f+3 d^{4} 5 f$. The appropriate computer program [16] calculates the angular part of the matrix element $\left\langle\phi S L J\left\|P^{1}\right\| \phi^{\prime} S^{\prime} L^{\prime} J^{\prime}\right\rangle$. From Eqs. (2) and (5), we can express the $g f$-values as a linear combination

$$
\begin{aligned}
(g f)^{1 / 2}= & \sum_{n l, n^{\prime} l^{\prime}}\left(303.76 \times 10^{-8} \sigma\right)^{1 / 2} \\
& \times \sum_{i} \sum_{j} c_{i} c_{j}^{\prime}\left\langle\phi_{i} S_{i} L_{i} J\left\|P^{1}\right\| \phi_{j}^{\prime} S_{j}^{\prime} L_{j}^{\prime} J^{\prime}\right\rangle
\end{aligned}
$$

where $\sigma$ is the wavenumber, given in $\mathrm{cm}^{-1}$, and the sum extends over all possible transitions ( $n \mathrm{~s} \leftrightarrow n^{\prime} \mathrm{p}, n \mathrm{~d} \leftrightarrow n^{\prime} \mathrm{p}, n \mathrm{~d} \leftrightarrow n^{\prime} \mathrm{f}$ ). The probability per unit time of an atom in a specific state $\gamma \mathrm{J}$ to make a spontaneous transition to any state with lower energy is $P(\gamma \mathrm{J})=$ $\sum A\left(\gamma J, \gamma^{\prime} J^{\prime}\right)$, where $A\left(\gamma J, \gamma^{\prime} J^{\prime}\right)$ is the Einstein spontaneous emission transition probability rate for a transition from $\gamma J$ to $\gamma^{\prime} J^{\prime}$ states. The sum is over all $\gamma^{\prime} J^{\prime}$ states with $E\left(\gamma^{\prime} J^{\prime}\right)<E(\gamma J)$.

The weighted transition probability is [17]

$g A=\left(2 J^{\prime}+1\right) A=\frac{64 \pi^{4} e^{2} a_{0}}{3 h} \sigma^{3} S=2.0261 \times 10^{-6} \sigma^{3} S$

where $\sigma$ is given, as previously, in $\mathrm{cm}^{-1}$ and $S$ in atomic units of $e^{2} a_{0}^{2}$. Substitution of Eq. (2) into (7) leads to

$\left(2 J^{\prime}+1\right) A=0.66702 \sigma^{2} g f$.

To determine the branching fractions it is necessary to measure the lifetime of the upper level in the case of emission or the lifetime of the lower level in the case of absorption, as well as the relative intensities of all lines originating from the considered level. The branching fraction, $B F$, is defined in the case of emission, appropriate to experimental data of interest here, as

$B F_{u l}=\frac{A_{u l}}{\sum_{k} A_{u k}}=\frac{I_{u l}}{\sum_{k} I_{u k}}$

with $u$ standing for the upper and $l$ the lower levels. The lifetime, $\tau$, is defined as the inverse of the probability

$\tau_{u}=\frac{1}{\sum_{k} A_{u k}}$.

Using Eqs. (9) and (10) one obtains

$B F_{u l}=A_{u l} \times \tau_{u}$

\subsection{Lifetime considerations}

Engman et al. [18] and Pinnington et al. [19] have used the beam-foil technique to measure radiative lifetimes of low-lying levels of the $\mathrm{Cr}$ II $3 \mathrm{~d}^{4}\left({ }^{5} \mathrm{D}\right) 4 \mathrm{p}$ configuration. Later Schade et al. [20], Pinnington et al. [14], Bergeson and Lawler [10] and Nilsson et al. [11] have extended these experimental data using the timeresolved laser-induced fluorescence method. The latter technique has been improved by the use of a frequency-doubled distributedfeedback dye laser which is directly pumped by a part of a XeClexcimer laser beam. These data are shown in Table 1.

\subsection{Oscillator strength determination}

To evaluate line strengths we have recourse to Eq. (5). As in previous works devoted to oscillator strength determination (see for instance [27]), the angular coefficients of the transition matrix, obtained in pure SL coupling with the help of Racah algebra is transformed into the actual intermediate coupling pertaining to our level eigenvector amplitudes, Moreover the transition integrals

$$
\int_{0}^{\infty} P_{n l}(r) r P_{n^{\prime} l^{\prime}}(r) d r
$$

are treated as free parameters in the least squares fit to experimental gf values. As we proceeded previously we have first sorted out the strongest lines, not blended, and particularly those representing transitions between levels with a limited number of leading components, displayed in their entirety for the reader in Tables 2 and 3 (up to $0.005 \%$ ). With the combination of time-resolved laser induced fluorescence radiative lifetime determinations and FTS branching fraction measurements, Nilsson et al. [11] have generated a complete set of $g f$-values in the wavelength range $2050-4850 \AA$ with an uncertainty varying from $3 \%$ to $42 \%$. From these $\mathrm{Cr}$ II $3 \mathrm{~d}^{4} 4 \mathrm{~s}-3 \mathrm{~d}^{4} 4 \mathrm{p}$ transitions we have selected in a first stage only those depopulating low-lying $3 \mathrm{~d}^{4} 4 \mathrm{p}$ levels (the highly excited levels will be considered in another paper). The comparison of 119 improved experimental [11] and calculated oscillator strengths of these selected transitions (including 94 fitted values) is presented in Table 4. In the latter, we additionally have inserted some other data found in the literature. For most transitions, the observed and calculated oscillator strength values are very consistent within the experimental accuracy. Then we have extracted semi-empirically, with a very good accuracy the transition radial integral values, reported in Table 5. In this table we have inserted also the transition radial integral value of $3 d^{4} 4 p-3 d^{4} 4 d$, admittedly with less good accuracy since we have rooted out only the $3 \mathrm{~d}^{4} 4 \mathrm{~d}$ contributions to $3 \mathrm{~d}^{4} 4 \mathrm{~s}$ levels. Let us point out that the obtained $\left\langle 3 \mathrm{~d}^{4} 4 \mathrm{~s}\left|r^{1}\right| 3 \mathrm{~d}^{4} 4 \mathrm{p}\right\rangle=$ $2.932(0.003)$ is very close to the predicted value reported in our previous work [9], i.e. $3.00(0.02)$. In the same table, we also give the results computed by means of the pseudo-relativistic HartreeFock (HFR) method using the basic Cowan code [17], on the one hand, and including core-polarization (HFR+CPOL), as described in $[21,22]$, on the other hand. As a reminder, in the latter approach, the radial dipole integrals given in Eq. (12) are replaced by

$$
\begin{aligned}
& \int_{0}^{\infty} P_{n l}(r) r\left[1-\frac{\alpha_{d}}{\left(r^{2}+r_{c}^{2}\right)^{3 / 2}}\right] P_{n^{\prime} l^{\prime}}(r) d r \\
& -\frac{\alpha_{d}}{r_{c}^{3}} \int_{0}^{r_{c}} P_{n l}(r) r P_{n^{\prime} l^{\prime}}(r) d r
\end{aligned}
$$

where $\alpha_{d}$ is the dipole polarizability of the ionic core, for which numerical values can be found in the literature (see e.g. [23]), and $r_{c}$ is the cut-off radius that is arbitrarily chosen as a measure of the size of the ionic core. In practice, this parameter is usually chosen to be equal to the HFR mean value $\langle r\rangle$ for the outermost ionic core orbital. In the present work, the HFR+CPOL approach was used with a Cr IV ionic core $\left(\alpha_{d}=\right.$ $1.96 a_{0}^{3}$ [23]; $\left.r_{c}=1.20 a_{0}\right)$ in addition to the explicit consideration of the following interacting configurations: $3 d^{5}, 3 d^{4} 4 s, 3 d^{4} 5 s$, $3 d^{4} 6 s, 3 d^{4} 4 d, 3 d^{4} 5 d, 3 d^{4} 6 d, 3 d^{3} 4 s^{2}, 3 d^{3} 4 p^{2}, 3 d^{3} 4 d^{2}, 3 d^{3} 4 s 4 d$, $3 d^{3} 4 s 5 s$ for the even parity, and $3 d^{4} 4 p, 3 d^{4} 5 p, 3 d^{4} 6 p, 3 d^{4} 4 f$, $3 d^{4} 5 f, 3 d^{4} 6 f, 3 d^{3} 4 s 4 p, 3 d^{3} 4 s 4 f, 3 d^{3} 4 p 4 d$ for the odd parity. Moreover, this HFR+CPOL model was combined with a wellestablished semi-empirical adjustment of the radial parameters in order to minimize the discrepancies between the calculated energy levels and the available experimental values [1-7] up to $105000 \mathrm{~cm}^{-1}$. In Table 1 , the radiative lifetimes obtained with this method are compared with the experimental data for the low-lying $3 d^{4} 4 p$ energy levels. As seen from this table a very good agreement is observed, the mean ratio $\tau_{H F R+C P O L} / \tau_{\text {exp }}$ 
resulting to $0.96 \pm 0.04$ when considering the two sets of experimental lifetime values due to Schade et al. [20] and Nilsson et al. [11].

\subsection{Transition probability and branching fraction derivations}

After having considered the radiative lifetimes, the oscillator strengths and transition radial integrals, we propose to close this study by computing the transition probabilities and branching fractions for all transitions depopulating the low-lying $3 \mathrm{~d}^{4} 4 \mathrm{p}$ levels (lower than $54785 \mathrm{~cm}^{-1}$ ) and whose $\log (g f) \geq-4$, using HFR + CPOL model and to compare them with semi-empirical data of Kurucz [24]. All these data are reported in Table 6 from which a rather fairly good agreement can be observed for transitions covering a wide wavelength range, from 2344 to $7580 \AA$ A.

\section{Conclusion}

We have extended our previous studies concerning oscillator strengths to another singly ionized atom: $\mathrm{Cr}$ II. In the whole our semi-empirical calculations agree well with experimental data within the uncertainty of measurements found in the literature. Our predictions for $\mathrm{Cr}$ II transition radial integral values have turned out favorably thanks to this work and now we can consider as a law what we previously consider only as general trends, seeing the large number of recognized cases: a decreasing of transition radial integral values with filling nd-shells of the same principal quantum numbers for $n \mathrm{~d}^{k}(n+1) \mathrm{s} \rightarrow n \mathrm{~d}^{k}(n+1) \mathrm{p}$ transitions. We will check again these values in the near future since we plan to compute the gf-values of transitions depopulating higher $\mathrm{Cr}$ II levels than those considered in the present work.

\section{Acknowledgments}

PQ and PP are respectively Research Director and Research Associate of the Belgian Fund for Scientific Research F.R.S.-FNRS. Financial support from this organization is deeply acknowledged.

\section{References}

[1] J. Sugar, C. Corliss, J. Phys. Chem. Ref. Data Suppl. 14 (1985) 264.

[2] C.C. Kiess, J. Res. Nat. Bur. Std. 60 (1958) 375 RP2856.

[3] S. Johansson, T. Zethson, H. Hartman, et al., Astron. Astrophys. 361 (2000) 977.

[4] C.J. Sansonetti, F. Kerber, J. Reader, M.R. Rosa, Astrophys. J. Suppl. Series 153 (2004) 555.

[5] C.J. Sansonetti, G. Nave, J. Reader, F. Kerber, Astrophys. J Suppl. Series 202 (2012) 15.

[6] C.J. Sansonetti, G. Nave, Astrophys. J. Suppl. Series 213 (2014) 1.

[7] E.B. Saloman, J. Phys. Chem. Ref. Data 41 (2012) 043103.

[8] S. Bouazza, P. Quinet, P. Palmeri, (ADNDT), accepted, http://dx.doi.org/10 1016/j.adt.201705003.

[9] S. Bouazza, P. Quinet, P. Palmeri, J. Quant. Spectrosc. Radiat. Transf. 199 (2017) 66.

[10] S.D. Bergeson, J.E. Lawler, Astrophys. J. 408 (1993) 382.

[11] H. Nilsson, G. Lyung, H. Lundberg, K.E. Nielsen, Astron. Astrophys. 445 (2006) 1165.

[12] J. Musielok, T. Wujec, Astron. Astrophys. Suppl. Series 38 (1979) 119.

[13] J. Gurell, H. Nilsson, L. Engström, H. Lundberg, R. Blackwell-Whitehead, K.E. Nielsen, S. Mannervik, Astron. Astrophys. 511 (2010) A68.

[14] E.H. Pinnington, Q. Ji, B. Gou, et al., Can. J. Phys. 71 (1993) 470.

[15] I. Sobelman, Atomic Spectra and Radiative Transitions, Springer-Verlag, Berlin, 1978.

[16] J. Ruczkowski, M. Elantkowska, J. Dembczynski, J. Quant. Spectrosc. Radiat. Transf. 145 (2014) 20.

[17] R.D. Cowan, The Theory of Atomic Structure Spectra, University of California Press, Berkeley, CA, 1981.

[18] B. Engman, A. Gaupp, L.J. Curtis, I. Martinson, Phys. Scr. 12 (1975) 220.

[19] E.H. Pinnington, H.O. Lutz, G.W. Carriaveao, Nucl. Instrum. Methods 110 (1973) 55.

[20] W. Schade, B. Mundt, V. Helbig, Phys. Rev. A 42 (1990) 3.

[21] P. Quinet, P. Palmeri, E. Biémont, M.M. McCurdy, G. Rieger, E.H. Pinnington, M.E. Wickliffe, J.E. Lawler, Mon. Not. R. Astron. Soc. 307 (1999) 934

[22] P. Quinet, P. Palmeri, E. Biémont, Z.S. Li, Z.G. Zhang, S. Svanberg, J. Alloys Compd. 344 (2002) 255.

[23] S. Fraga, J. Karwowski, K.M.S. Saxena, Handbook of Atomic Data, Elsevier Scientific Publishing Company, Amsterdam, 1976.

[24] R.L. Kurucz (1995), Atomic spectral line data from CD-ROM Nr. 23, http://www cfa.harvard.edu/amp/ampdata/kurucz23/sekur.html.

[25] R. Spreger, B. Schelm, M. Kock, T. Neger, M. Ulbel, J. Quant. Spectrosc. Radiat. Transf. 51 (1994) 779.

[26] A.M. Gonzalez, M. Ortiz, J. Compos, Can. J. Phys. 72 (1994) 57.

[27] J. Ruczkowski, S. Bouazza, M. Elantkowska, J. Dembczynski, J. Quant. Spectrosc. Radiat. Transf. 155 (2015) 1. 


\section{Explanation of Tables}

Table 1. Comparison between HFR+CPOL radiative lifetimes (in ns) and available experimental data for low-lying $3 \mathrm{~d}^{4} 4 \mathrm{p}$ energy levels of Cr II.

Level: $\quad$ Designation of the state in LSJ coupling within the configuration and where $J$ represents the total angular momentum.

Energy: $\quad$ Observed (experimental) energy level value in $\mathrm{cm}^{-1}$.

*HFR+CPOL: $\quad$ Computed lifetime (in ns) using Hartree-Fock method with relativistic corrections (HFR) implemented in the code developed by Cowan [17] and including core polarization (CPOL).

Experiment: $\quad$ Measured lifetimes (ns) obtained by beam-foil spectroscopy or laser induced fluorescence techniques.

Table 2. Eigenvalue components of even-parity $\mathrm{Cr}$ II levels of interest in this study.

Level: $\quad$ Configuration to which belongs the even-parity level involved in the studied transitions and the designation of this level in LS coupling.

J: $\quad$ Total angular momentum.

Energy: $\quad$ Experimental energy level $\left(\mathrm{cm}^{-1}\right)$.

$3 \mathrm{~d}^{5}: \quad$ Contribution in LS coupling of $3 \mathrm{~d}^{5}$ configuration (in \%) to the total composition of the level.

$3 \mathrm{~d}^{4} 4 \mathrm{~s}$ : $\quad$ Contribution in LS coupling of $3 \mathrm{~d}^{4} 4 \mathrm{~s}$ configuration (in \%) to the total composition of the level.

$3 \mathrm{~d}^{4} 4 \mathrm{~d}$ : $\quad$ Contribution in LS coupling of $3 \mathrm{~d}^{4} 4 \mathrm{~d}$ configuration (in \%) to the total composition of the level.

$3 \mathrm{~d}^{4} 5 \mathrm{~s}$ : $\quad$ Contribution in LS coupling of $3 \mathrm{~d}^{4} 5 \mathrm{~s}$ configuration (in \%) to the total composition of the level.

$3 \mathrm{~d}^{4} 6 \mathrm{~s}$ : $\quad$ Contribution in LS coupling of $3 \mathrm{~d}^{4} 6 \mathrm{~s}$ configuration (in \%) to the total composition of the level.

$3 \mathrm{~d}^{4} 5 \mathrm{~d}$ : $\quad$ Contribution in LS coupling of $3 \mathrm{~d}^{4} 5 \mathrm{~d}$ configuration (in \%) to the total composition of the level.

$3 \mathrm{~d}^{4} 6 \mathrm{~d}$ : $\quad$ Contribution in LS coupling of $3 \mathrm{~d}^{4} 6 \mathrm{~d}$ configuration (in \%) to the total composition of the level.

$3 \mathrm{~d}^{3} 4 \mathrm{~s}^{2}$ : $\quad$ Contribution in LS coupling of $3 \mathrm{~d}^{3} 4 \mathrm{~s}^{2}$ configuration (in \%) to the total composition of the level.

Table 3. Eigenvalue components of odd-parity $\mathrm{Cr}$ II levels of interest in this study.

Level:

Configuration to which belongs the odd-parity level involved in the studied transitions and the designation of this level in

LS coupling.

$J: \quad$ Total angular momentum.

Energy: $\quad$ Experimental energy level $\left(\mathrm{cm}^{-1}\right)$.

$3 \mathrm{~d}^{4} 4 \mathrm{p}$ : $\quad$ Contribution in LS coupling of $3 \mathrm{~d}^{4} 4 \mathrm{p}$ configuration (in \%) to the total composition of the level.

$3 \mathrm{~d}^{3} 4 \mathrm{~s} 4 \mathrm{p}$ : $\quad$ Contribution in LS coupling of $3 \mathrm{~d}^{3} 4 \mathrm{~s} 4 \mathrm{p}$ configuration (in \%) to the total composition of the level.

3d $3 \mathrm{~d}^{4} \mathrm{p}$ : Contribution in LS coupling of $3 \mathrm{~d}^{4} 5 \mathrm{p}$ configuration (in \%) to the total composition of the level.

$3 \mathrm{~d}^{4} 6 \mathrm{p}$ : Contribution in LS coupling of $3 \mathrm{~d}^{4} 6 \mathrm{p}$ configuration (in \%) to the total composition of the level.

Table 4. Comparison between experimental and calculated oscillator strength values.

$\lambda_{\text {air }}$ : $\quad$ Wavelength in air of the spectral line $(\AA)$ deduced from observed energy levels taken from [11].

Upper Level: $\quad$ Depopulated upper level $[11]\left(\mathrm{cm}^{-1}\right)$ in emission transition.

$J(\mathrm{U}): \quad \quad$ Total angular momentum of the upper level.

Lower Level: $\quad$ Populated lower level $[11]\left(\mathrm{cm}^{-1}\right)$ in emission transition.

$J(\mathrm{~L})$ :

Total angular momentum of the lower level.

gf(exp):

$\Delta g f(\exp ):$

Measured weighted oscillator strength value

gf(calc):

Experimental uncertainty (\%) for measured weighted oscillator strength value from [11].

gf [11](dimensionless). Here OSP means (oscillator strength parameterisation)

$\log (g f): \quad \quad$ Weighted oscillator strength in logarithmic scale from this work (OSP) and other studies [10-12,24-26].

Table 5. Transition radial integral values in $\mathrm{Cr}$ II.

Transition: $\quad$ Spectral line linking levels of mentioned configurations.

HFR: $\quad$ Relativistic Hartree-Fock method.

HFR+CPOL: $\quad$ Relativistic Hartree-Fock method including core-polarization.

OSP: $\quad$ Oscillator Strength Parameterization method.

Table 6. Semi-empirical radiative data in $\mathrm{Cr}$ II.

Wavelength: $\quad$ Wavelength in Å of experimentally observed spectral lines.

E(Lower): $\quad$ Energy in $\mathrm{cm}^{-1}$ of lower level of the transition.

$\begin{array}{ll}\text { Parity (Lower): } & \text { Parity of lower level of the transition. 'e' and 'o' stand respectively for even and odd. } \\ J \text { (Lower): } & \text { Total angular momentum of the lower level of the transition. }\end{array}$

$J$ (Lower):

E(Upper):

Parity (Upper):

$J($ Upper):

Energy in $\mathrm{cm}^{-1}$ of upper level of the transition.

$\log (g f)($ HFR+CPOL):

$\log (g f)($ Kurucz):

$g A(\mathrm{HFR}+\mathrm{CPOL}):$

Parity of upper level of the transition. 'e' and 'o' stand respectively for even and odd.

Total angular momentum of the upper level of the transition.

HFR+CPOL weighted oscillator strength in logarithmic scale.

Weighted oscillator strength in the logarithmic scale from the Kurucz database [24].

$B F(\mathrm{HFR}+\mathrm{CPOL})$ :

HFR + CPOL weighted transition probability $\left(\mathrm{s}^{-1}\right)$.

HFR+CPOL branching fraction. 
Table 1

Comparison between HFR+CPOL radiative lifetimes (in ns) and available experimental data for low-lying $3 \mathrm{~d}^{4} 4 \mathrm{p}$ energy levels of $\mathrm{Cr}$ II.

\begin{tabular}{|c|c|c|c|c|c|c|c|}
\hline \multirow[t]{2}{*}{ Level } & \multirow{2}{*}{$\begin{array}{l}\text { Energy } \\
\left(\mathrm{cm}^{-1}\right)\end{array}$} & \multirow[t]{2}{*}{$\mathrm{HFR}+\mathrm{CPOL}$} & \multicolumn{5}{|c|}{ Experiment } \\
\hline & & & Ref. [11] & Ref. [20] & Refs. [14,19] & Ref. [10] & Ref. [18] \\
\hline${ }^{6} \mathrm{~F}_{1 / 2}$ & 46824 & 4.2 & & $4.3(1)$ & & & \\
\hline${ }^{6} \mathrm{~F}_{3 / 2}$ & 46906 & 4.1 & & $4.2(1)$ & & & \\
\hline${ }^{6} \mathrm{~F}_{5 / 2}$ & 47041 & 4.1 & & $4.2(1)$ & & & \\
\hline${ }^{6} \mathrm{~F}_{7 / 2}$ & 47228 & 4.1 & & $4.1(1)$ & & & \\
\hline${ }^{6} \mathrm{~F}_{9 / 2}$ & 47465 & 4.1 & & $4.2(1)$ & & & \\
\hline${ }^{6} \mathrm{~F}_{11 / 2}$ & 47752 & 4.0 & & $4.0(1)$ & & & \\
\hline${ }^{6} \mathrm{P}_{3 / 2}$ & 48399 & 2.2 & & $2.4(1)$ & & & \\
\hline${ }^{6} \mathrm{P}_{5 / 2}$ & 48491 & 2.2 & $2.3(2)$ & $2.5(1)$ & 2.45 & $2.4(2)$ & $3.2(4)$ \\
\hline${ }^{6} \mathrm{P}_{7 / 2}$ & 48632 & 2.2 & $2.4(2)$ & $2.5(1)$ & $2.40(13)$ & $2.4(2)$ & $3.3(4)$ \\
\hline${ }^{4} \mathrm{P}_{1 / 2}$ & 48750 & 4.8 & & $5.0(2)$ & & & \\
\hline${ }^{4} \mathrm{P}_{3 / 2}$ & 49006 & 4.7 & & $4.7(2)$ & & & \\
\hline${ }^{4} \mathrm{P}_{5 / 2}$ & 49352 & 4.3 & & $4.6(2)$ & & & \\
\hline${ }^{6} \mathrm{D}_{1 / 2}$ & 49493 & 3.8 & & $4.3(2)$ & & & \\
\hline${ }^{6} \mathrm{D}_{3 / 2}$ & 49565 & 3.9 & & $4.2(1)$ & & & \\
\hline${ }^{6} \mathrm{D}_{7 / 2}$ & 49646 & 3.6 & & $3.8(1)$ & & & \\
\hline${ }^{6} \mathrm{D}_{5 / 2}$ & 49706 & 4.1 & & $4.5(1)$ & & & \\
\hline${ }^{6} \mathrm{D}_{9 / 2}$ & 49838 & 3.6 & & $3.8(2)$ & & & \\
\hline${ }^{4} \mathrm{~F}_{3 / 2}$ & 51584 & 4.2 & $4.2(4)$ & & & & \\
\hline${ }^{4} \mathrm{~F}_{5 / 2}$ & 51669 & 4.2 & $4.1(4)$ & & & & \\
\hline${ }^{4} \mathrm{~F}_{7 / 2}$ & 51789 & 4.2 & $4.1(3)$ & & & & \\
\hline${ }^{4} \mathrm{~F}_{9 / 2}$ & 51943 & 4.2 & $4.1(3)$ & & & & \\
\hline${ }^{4} \mathrm{D}_{1 / 2}$ & 54418 & 4.1 & $4.3(4)$ & & & & \\
\hline${ }^{4} \mathrm{D}_{3 / 2}$ & 54500 & 4.1 & $4.3(4)$ & & & & \\
\hline${ }^{4} D_{5 / 2}$ & 54626 & 4.1 & $4.3(4)$ & & & & \\
\hline${ }^{4} \mathrm{D}_{5 / 2}$ & 54784 & 4.1 & $4.3(4)$ & & $4.20(18)$ & & \\
\hline
\end{tabular}

Table 2

Eigenvalue components of even-parity $\mathrm{Cr}$ II levels of interest in this study.

\begin{tabular}{|c|c|c|c|c|c|c|c|c|c|c|}
\hline Level & $J$ & $\begin{array}{l}\text { Energy } \\
\left(\mathrm{cm}^{-1}\right)\end{array}$ & $\begin{array}{l}3 d^{5} \\
(\%)\end{array}$ & $\begin{array}{l}3 d^{4} 4 s \\
(\%)\end{array}$ & $\begin{array}{l}3 d^{4} 4 d \\
(\%)\end{array}$ & $\begin{array}{l}3 d^{4} 5 s \\
(\%)\end{array}$ & $\begin{array}{l}3 d^{4} 6 s \\
(\%)\end{array}$ & $\begin{array}{l}3 d^{4} 5 d \\
(\%)\end{array}$ & $\begin{array}{l}3 d^{4} 6 d \\
(\%)\end{array}$ & $\begin{array}{l}3 d^{3} 4 s^{2} \\
(\%)\end{array}$ \\
\hline $3 d^{4} 4 s a^{6} D$ & $1 / 2$ & 11962 & & 99.93 & 0.02 & 0.03 & & & & \\
\hline $3 d^{4} 4 s a^{4} D$ & $1 / 2$ & 19528 & 4.36 & 95.35 & 0.16 & 0.02 & 0.03 & 0.01 & & \\
\hline $3 d^{5} a^{4} P$ & $1 / 2$ & 21824 & 97.21 & 1.39 & 0.69 & 0.02 & & 0.17 & 0.06 & 0.43 \\
\hline $3 d^{4} 4 s a^{6} D$ & $3 / 2$ & 12033 & & 99.93 & 0.02 & 0.03 & & & & \\
\hline $3 d^{4} 4 s a^{4} D$ & $3 / 2$ & 19631 & 4.36 & 95.08 & 0.16 & 0.06 & 0.02 & 0.03 & 0.01 & \\
\hline $3 d^{5} a^{4} P$ & $3 / 2$ & 21824 & 97.21 & 1.43 & 0.69 & 0.02 & & 0.17 & 0.06 & 0.42 \\
\hline $3 d^{5} a^{6} S$ & $5 / 2$ & 0 & 99.91 & & 0.01 & & & 0.06 & 0.01 & \\
\hline $3 d^{4} 4 s a^{6} D$ & $5 / 2$ & 12148 & & 99.93 & 0.02 & 0.03 & & & & \\
\hline $3 d^{4} 4 s a^{4} D$ & $5 / 2$ & 19798 & 4.99 & 94.72 & 0.17 & 0.06 & 0.02 & 0.03 & 0.01 & \\
\hline $3 d^{5} a^{4} G$ & $5 / 2$ & 20512 & 98.99 & 0.40 & 0.39 & 0.01 & & 0.16 & 0.05 & \\
\hline $3 d^{5} a^{4} P$ & $5 / 2$ & 21823 & 97.26 & 1.39 & 0.69 & 0.02 & & 0.17 & & 0.42 \\
\hline $3 d^{4} 4 s a^{6} D$ & $7 / 2$ & 12304 & & 99.93 & 0.02 & 0.03 & & & & \\
\hline $3 d^{4} 4 s a^{4} D$ & $7 / 2$ & 20024 & 5.17 & 94.53 & 0.17 & 0.06 & 0.02 & 0.03 & 0.01 & \\
\hline $3 d^{5} a^{4} G$ & $7 / 2$ & 20518 & 99.00 & 0.40 & 0.39 & 0.01 & & 0.15 & 0.05 & \\
\hline $3 d^{4} 4 s a^{6} D$ & $9 / 2$ & 12496 & & 99.93 & 0.02 & 0.03 & & & & \\
\hline $3 d^{5} a^{4} G$ & $11 / 2$ & 20512 & 99.02 & 0.38 & 0.38 & 0.01 & & 0.15 & 0.05 & \\
\hline
\end{tabular}


Table 3

Eigenvalue components of odd-parity $\mathrm{Cr}$ II levels of interest in this study.

\begin{tabular}{|c|c|c|c|c|c|c|}
\hline Level & $J$ & $\begin{array}{l}\text { Energy } \\
\left(\mathrm{cm}^{-1}\right)\end{array}$ & $\begin{array}{l}3 d^{4} 4 p \\
(\%)\end{array}$ & $\begin{array}{l}3 d^{3} 4 s 4 p \\
(\%)\end{array}$ & $\begin{array}{l}3 d^{4} 5 p \\
(\%)\end{array}$ & $\begin{array}{l}3 d^{4} 6 p \\
(\%)\end{array}$ \\
\hline$z^{6} F$ & $1 / 2$ & 46823 & 99.57 & 0.10 & 0.27 & 0.06 \\
\hline $\mathrm{z}^{4} \mathrm{P}$ & $1 / 2$ & 48749 & 99.62 & 0.22 & 0.12 & 0.03 \\
\hline$z^{6} D$ & $1 / 2$ & 49493 & 99.52 & 0.31 & 0.13 & 0.02 \\
\hline$z^{4} D$ & $1 / 2$ & 54418 & 99.48 & 0.37 & 0.11 & 0.03 \\
\hline $\mathrm{z}^{6} \mathrm{~F}$ & $3 / 2$ & 46905 & 99.57 & 0.10 & 0.27 & 0.06 \\
\hline$z^{6} P$ & $3 / 2$ & 48399 & 99.74 & 0.13 & 0.09 & 0.03 \\
\hline $\mathrm{z}^{4} \mathrm{P}$ & $3 / 2$ & 49006 & 99.62 & 0.23 & 0.12 & 0.03 \\
\hline$z^{6} D$ & $3 / 2$ & 49565 & 99.52 & 0.31 & 0.13 & 0.02 \\
\hline$z^{4} D$ & $3 / 2$ & 54499 & 99.49 & 0.37 & 0.11 & 0.03 \\
\hline$z^{6} F$ & $5 / 2$ & 47040 & 99.57 & 0.10 & 0.27 & 0.06 \\
\hline$z^{6} P$ & $5 / 2$ & 48391 & 99.74 & 0.13 & 0.09 & 0.03 \\
\hline$z^{6} D$ & $5 / 2$ & 49352 & 99.59 & 0.26 & 0.13 & 0.03 \\
\hline $\mathrm{z}^{4} \mathrm{P}$ & $5 / 2$ & 49706 & 99.56 & 0.28 & 0.13 & 0.02 \\
\hline $\mathrm{z}^{6} \mathrm{~F}$ & $7 / 2$ & 47227 & 99.57 & 0.10 & 0.27 & 0.06 \\
\hline$z^{6} P$ & $7 / 2$ & 48632 & 99.74 & 0.13 & 0.09 & 0.03 \\
\hline$z^{6} D$ & $7 / 2$ & 49646 & 99.49 & 0.35 & 0.14 & 0.02 \\
\hline $\mathrm{z}^{4} \mathrm{~F}$ & $7 / 2$ & 51789 & 99.74 & 0.14 & 0.09 & 0.02 \\
\hline$z^{4} D$ & $7 / 2$ & 54784 & 99.49 & 0.36 & 0.11 & 0.03 \\
\hline$z^{6} F$ & $9 / 2$ & 47465 & 99.57 & 0.10 & 0.27 & 0.06 \\
\hline$z^{6} D$ & $9 / 2$ & 49838 & 99.49 & 0.34 & 0.14 & 0.02 \\
\hline $\mathrm{z}^{4} \mathrm{~F}$ & $9 / 2$ & 51943 & 99.74 & 0.14 & 0.09 & 0.02 \\
\hline
\end{tabular}


Table 4

\begin{tabular}{|c|c|c|c|c|c|c|c|c|c|c|c|c|c|c|}
\hline \multirow{2}{*}{$\begin{array}{l}\lambda_{\text {air }} \\
(\AA)\end{array}$} & \multirow{2}{*}{$\begin{array}{l}\text { Upper Level } \\
\left(\mathrm{cm}^{-1}\right)\end{array}$} & \multirow[t]{2}{*}{$J(\mathrm{U})$} & \multirow{2}{*}{$\begin{array}{l}\text { Lower Level } \\
\left(\mathrm{cm}^{-1}\right)\end{array}$} & \multirow[t]{2}{*}{$J(\mathrm{~L})$} & \multirow[t]{2}{*}{$g f(\exp )$} & \multirow{2}{*}{\multicolumn{2}{|c|}{$\begin{array}{l}\Delta g f(\exp ) g f(\text { calc }) \\
(\%)\end{array}$}} & \multicolumn{7}{|l|}{$\log (g f)$} \\
\hline & & & & & & & & [11] & OSP & [24] & [25] & [10] & [26] & [12] \\
\hline 2531.85 & 51788.816 & $7 / 2$ & 12303.82 & $7 / 2$ & 0.0182 & 12 & 0.0106 & $\begin{array}{l}-1.740 \\
\end{array}$ & -1.9756 & $\begin{array}{l}-1.878 \\
\end{array}$ & & & & \\
\hline 2534.33 & 51942.664 & $9 / 2$ & 12496.456 & $9 / 2$ & 0.0484 & 12 & 0.0345 & -1.315 & -1.4616 & -1.361 & & & & \\
\hline 2653.58 & 49706.262 & $5 / 2$ & 12032.545 & $3 / 2$ & 0.2415 & 6 & 0.4034 & -0.617 & -0.3943 & -0.304 & & & & \\
\hline 2658.59 & 49564.504 & $3 / 2$ & 11961.747 & $1 / 2$ & 0.2642 & 8 & 0.3447 & -0.578 & -0.4626 & -0.45 & & & & \\
\hline 2661.72 & 49706.262 & $5 / 2$ & 12147.772 & $5 / 2$ & 0.0728 & 6 & 0.1355 & -1.138 & -0.8681 & -0.755 & & & & \\
\hline 2663.42 & 49838.379 & $9 / 2$ & 12303.82 & $7 / 2$ & 0.561 & 40 & 0.633 & -0.251 & -0.1986 & -0.205 & & & & \\
\hline 2663.60 & 49564.504 & $3 / 2$ & 12032.545 & $3 / 2$ & 0.0238 & 9 & 0.0049 & -1.623 & -2.3056 & -2.181 & & & & \\
\hline 2663.67 & 49492.711 & $1 / 2$ & 11961.747 & $1 / 2$ & 0.0912 & 8 & 0.1127 & -1.040 & -0.9480 & -0.973 & & & & \\
\hline 2666.01 & 49645.805 & $7 / 2$ & 12147.772 & $5 / 2$ & 0.7834 & 23 & 0.8761 & -0.106 & -0.0574 & -0.064 & -0.1 & & -0.056 & -0.313 \\
\hline 2668.71 & 49492.711 & $1 / 2$ & 12032.545 & $3 / 2$ & 0.3097 & 8 & 0.3863 & -0.509 & -0.4131 & -0.448 & -0.67 & & -0.537 & -0.557 \\
\hline 2671.81 & 49564.504 & $3 / 2$ & 12147.772 & $5 / 2$ & 0.4667 & 7 & 0.5881 & -0.331 & -0.2306 & -0.271 & -0.34 & & -0.373 & -0.377 \\
\hline 2672.83 & 49706.262 & $5 / 2$ & 12303.82 & $7 / 2$ & 0.369 & 6 & 0.5366 & -0.433 & -0.2703 & -0.281 & -0.59 & & & -0.468 \\
\hline 2678.79 & 49351.734 & $5 / 2$ & 12032.545 & $3 / 2$ & 0.5176 & 7 & 0.4382 & -0.286 & -0.3583 & -0.475 & -0.39 & & -0.351 & \\
\hline 2687.09 & 49351.734 & $5 / 2$ & 12147.772 & $5 / 2$ & 0.2786 & 7 & 0.2565 & -0.555 & -0.5908 & -0.674 & -0.6 & & -0.608 & \\
\hline 2691.04 & 49645.805 & $7 / 2$ & 12496.456 & $9 / 2$ & 0.4446 & 23 & 0.5079 & -0.352 & -0.2942 & -0.403 & & & -0.337 & \\
\hline 2698.41 & 49351.734 & $5 / 2$ & 12303.82 & $7 / 2$ & 0.2871 & 8 & 0.1931 & -0.542 & -0.7143 & -1.077 & & & & \\
\hline 2698.68 & 49005.848 & $3 / 2$ & 11961.747 & $1 / 2$ & 0.2729 & 6 & 0.2362 & -0.564 & -0.6268 & -0.656 & & & & \\
\hline 2703.85 & 49005.848 & $3 / 2$ & 12032.545 & $3 / 2$ & 0.0406 & 7 & 0.043 & -1.392 & -1.3664 & -1.327 & & & & \\
\hline 2712.31 & 49005.848 & $3 / 2$ & 12147.772 & $5 / 2$ & 0.159 & 6 & 0.106 & -0.799 & -0.9746 & -1.167 & -0.76 & & & \\
\hline 2717.51 & 48749.277 & $1 / 2$ & 11961.747 & $1 / 2$ & 0.0372 & 8 & 0.0308 & -1.430 & -1.5121 & -1.543 & & & & \\
\hline 2722.75 & 48749.277 & $1 / 2$ & 12032.545 & $3 / 2$ & 0.1361 & 6 & 0.1077 & -0.866 & -0.9678 & -1.014 & -1.002 & & & \\
\hline 2740.10 & 48632.059 & $7 / 2$ & 12147.772 & $5 / 2$ & 0.0798 & 12 & 0.081 & -1.098 & -1.0917 & -1.233 & -1.18 & -1.09 & -1.091 & -1.004 \\
\hline 2742.03 & 48491.059 & $5 / 2$ & 12032.545 & $3 / 2$ & 0.2042 & 9 & 0.1945 & -0.690 & -0.7111 & -0.817 & -0.82 & -0.68 & -0.626 & \\
\hline 2743.64 & 48398.871 & $3 / 2$ & 11961.747 & $1 / 2$ & 0.3459 & 9 & 0.3349 & -0.461 & -0.4751 & -0.545 & & -0.47 & -0.47 & \\
\hline 2748.98 & 48398.871 & $3 / 2$ & 12032.545 & $3 / 2$ & 0.527 & 9 & 0.5378 & -0.278 & -0.2694 & -0.305 & -0.52 & -0.28 & -0.257 & \\
\hline 2750.73 & 48491.059 & $5 / 2$ & 12147.772 & $5 / 2$ & 0.6501 & 9 & 0.6714 & -0.187 & -0.1730 & -0.226 & -0.43 & -0.18 & -0.199 & \\
\hline 2751.86 & 48632.059 & $7 / 2$ & 12303.82 & $7 / 2$ & 0.5058 & 9 & 0.5286 & -0.296 & -0.2769 & -0.349 & -0.69 & -0.29 & -0.294 & \\
\hline 2757.73 & 48398.871 & $3 / 2$ & 12147.772 & $5 / 2$ & 0.455 & 9 & 0.4463 & -0.342 & -0.3504 & -0.349 & -0.53 & -0.36 & -0.372 & \\
\hline 2762.59 & 48491.059 & $5 / 2$ & 12303.82 & $7 / 2$ & 1.0914 & 9 & 1.1292 & 0.038 & 0.0528 & 0.048 & -0.28 & 0.05 & 0.024 & \\
\hline 2766.53 & 48632.059 & $7 / 2$ & 12496.456 & $9 / 2$ & 2.0512 & 9 & 2.1053 & 0.312 & 0.3233 & 0.31 & & 0.32 & 0.301 & \\
\hline 2835.63 & 47751.602 & $11 / 2$ & 12496.456 & $9 / 2$ & 3.6141 & 3 & 3.8391 & 0.558 & 0.5842 & 0.572 & & & 0.562 & \\
\hline 2849.83 & 47227.219 & $7 / 2$ & 12147.772 & $5 / 2$ & 1.4852 & 3 & 1.6041 & 0.171 & 0.2052 & 0.184 & 0.13 & & 0.15 & -0.067 \\
\hline 2855.67 & 47040.273 & $5 / 2$ & 12032.545 & $3 / 2$ & 0.8299 & 4 & 0.8964 & -0.081 & -0.0475 & -0.069 & -0.18 & & -0.101 & \\
\hline 2856.76 & 54625.594 & $5 / 2$ & 19631.205 & $3 / 2$ & 0.241 & 10 & 0.3301 & -0.618 & -0.4813 & -0.598 & -0.71 & & -0.511 & \\
\hline 2857.40 & 54784.449 & $7 / 2$ & 19797.859 & $5 / 2$ & 0.2061 & 11 & 0.2549 & -0.686 & -0.5937 & -0.698 & & & -0.562 & \\
\hline 2858.65 & 54499.492 & $3 / 2$ & 19528.23 & $1 / 2$ & 0.1832 & 11 & 0.25 & -0.737 & -0.6021 & -0.726 & & & & \\
\hline 2858.91 & 47464.559 & $9 / 2$ & 12496.456 & $9 / 2$ & 0.597 & 6 & 0.6208 & -0.224 & -0.2071 & -0.196 & & & -0.179 & \\
\hline 2860.93 & 46905.137 & $3 / 2$ & 11961.747 & $1 / 2$ & 0.3556 & 5 & 0.3882 & -0.449 & -0.4110 & -0.432 & & & -0.503 & -0.49 \\
\hline 2862.57 & 47227.219 & $7 / 2$ & 12303.82 & $7 / 2$ & 0.8511 & 5 & 0.8844 & -0.070 & -0.0534 & -0.053 & -0.45 & -0.034 & -0.229 & \\
\hline 2865.10 & 47040.273 & $5 / 2$ & 12147.772 & $5 / 2$ & 0.8166 & 4 & 0.8906 & -0.088 & -0.0503 & -0.057 & -0.12 & & -0.045 & \\
\hline 2865.33 & 54417.957 & $1 / 2$ & 19528.23 & $1 / 2$ & 0.1901 & 11 & 0.2799 & -0.721 & -0.5530 & -0.688 & & & & \\
\hline 2867.09 & 54499.492 & $3 / 2$ & 19631.205 & $3 / 2$ & 0.3251 & 10 & 0.4636 & -0.488 & -0.3339 & -0.474 & & & & -0.266 \\
\hline 2867.65 & 46823.305 & $1 / 2$ & 11961.747 & $1 / 2$ & 0.4365 & 3 & 0.4902 & -0.360 & -0.3096 & -0.324 & & & -0.353 & -0.567 \\
\hline 2873.48 & 46823.305 & $1 / 2$ & 12032.545 & $3 / 2$ & 0.138 & 6 & 0.1417 & -0.860 & -0.8486 & -0.853 & & & -0.843 & \\
\hline 2873.81 & 54417.957 & $1 / 2$ & 19631.205 & $3 / 2$ & 0.2104 & 11 & 0.2965 & -0.677 & -0.5279 & -0.662 & & & & -0.672 \\
\hline 2875.99 & 54784.449 & $7 / 2$ & 20024.012 & $7 / 2$ & 1.531 & 10 & 2.0221 & 0.185 & 0.3058 & 0.175 & & & & \\
\hline 2876.24 & 46905.137 & $3 / 2$ & 12147.772 & $5 / 2$ & 0.1462 & 5 & 0.155 & -0.835 & -0.8096 & -0.804 & & & -0.58 & \\
\hline 2878.44 & 47227.219 & $7 / 2$ & 12496.456 & $9 / 2$ & 0.0481 & 8 & 0.0499 & -1.318 & -1.3016 & -1.27 & & & -1.304 & -1.158 \\
\hline 2880.86 & 54499.492 & $3 / 2$ & 19797.859 & $5 / 2$ & 0.317 & 10 & 0.4369 & -0.499 & -0.3596 & -0.493 & & & & -0.411 \\
\hline 2889.19 & 54625.594 & $5 / 2$ & 20024.012 & $7 / 2$ & 0.278 & 10 & 0.3752 & -0.556 & -0.4258 & -0.554 & & & & \\
\hline 3032.92 & 54784.449 & $7 / 2$ & 21822.506 & $5 / 2$ & 0.0486 & 20 & 0.0959 & -1.313 & -1.0183 & -1.031 & & & & \\
\hline 3047.61 & 54625.594 & $5 / 2$ & 21824.141 & $3 / 2$ & 0.0263 & 21 & 0.0535 & -1.580 & -1.2717 & -1.326 & & & & \\
\hline 3118.65 & 51584.102 & $3 / 2$ & 19528.23 & $1 / 2$ & 0.7907 & 10 & 0.6897 & -0.102 & -0.1613 & -0.081 & & & & -0.004 \\
\hline
\end{tabular}


Table 4 (continued)

\begin{tabular}{|c|c|c|c|c|c|c|c|c|c|c|c|c|c|c|}
\hline \multirow{2}{*}{$\begin{array}{l}\lambda_{\text {air }} \\
(\AA)\end{array}$} & \multirow{2}{*}{$\begin{array}{l}\text { Upper Level } \\
\left(\mathrm{cm}^{-1}\right)\end{array}$} & \multirow[t]{2}{*}{$J(\mathrm{U})$} & \multirow{2}{*}{$\begin{array}{l}\text { Lower Level } \\
\left(\mathrm{cm}^{-1}\right)\end{array}$} & \multirow[t]{2}{*}{$J(\mathrm{~L})$} & \multirow[t]{2}{*}{$g f(\exp )$} & \multirow{2}{*}{\multicolumn{2}{|c|}{$\begin{array}{l}\Delta g f(\exp ) g f(\text { calc }) \\
(\%)\end{array}$}} & \multicolumn{7}{|l|}{$\log (g f)$} \\
\hline & & & & & & & & [11] & OSP & [24] & [25] & [10] & [26] & [12] \\
\hline 3124.98 & 51788.816 & $7 / 2$ & 19797.859 & $5 / 2$ & 1.932 & 8 & 1.6578 & 0.286 & 0.2195 & 0.303 & 0.44 & & & \\
\hline 3128.70 & 51584.102 & $3 / 2$ & 19631.205 & $3 / 2$ & 0.2965 & 11 & 0.2452 & -0.528 & -0.6105 & -0.511 & -0.82 & & & -0.362 \\
\hline 3132.05 & 51942.664 & $9 / 2$ & 20024.012 & $7 / 2$ & 2.7353 & 7 & 2.308 & 0.437 & 0.3632 & 0.451 & 0.57 & & & \\
\hline 3136.69 & 51669.406 & $5 / 2$ & 19797.859 & $5 / 2$ & 0.3855 & 11 & 0.2978 & -0.414 & -0.5260 & -0.416 & -0.29 & & & -0.254 \\
\hline 3147.22 & 51788.816 & $7 / 2$ & 20024.012 & $7 / 2$ & 0.2965 & 9 & 0.2087 & -0.528 & -0.6805 & -0.558 & -0.18 & & & \\
\hline 3180.69 & 51942.664 & $9 / 2$ & 20512.098 & $11 / 2$ & 0.6383 & 9 & 0.4372 & -0.195 & -0.3593 & -0.131 & & & & \\
\hline 3209.18 & 51669.406 & $5 / 2$ & 20517.793 & $7 / 2$ & 0.3882 & 11 & 0.2476 & -0.411 & -0.6063 & -0.372 & & & & -0.214 \\
\hline 3217.40 & 51584.102 & $3 / 2$ & 20512.062 & $5 / 2$ & 0.2704 & 11 & 0.1831 & -0.568 & -0.7373 & -0.501 & & & & -0.332 \\
\hline 3324.06 & 49706.262 & $5 / 2$ & 19631.205 & $3 / 2$ & 0.0296 & 14 & 0.0216 & -1.529 & -1.6648 & -1.691 & -1.21 & & & \\
\hline 3328.35 & 49564.504 & $3 / 2$ & 19528.23 & $1 / 2$ & 0.0196 & 22 & 0.0156 & -1.708 & -1.8067 & -1.752 & & & & \\
\hline 3336.33 & 49492.711 & $1 / 2$ & 19528.23 & $1 / 2$ & 0.0673 & 14 & 0.0495 & -1.172 & -1.3054 & -1.172 & & & & \\
\hline 3339.80 & 49564.504 & $3 / 2$ & 19631.205 & $3 / 2$ & 0.1057 & 13 & 0.0762 & -0.976 & -1.1182 & -1.044 & -1.03 & & & \\
\hline 3342.58 & 49706.262 & $5 / 2$ & 19797.859 & $5 / 2$ & 0.1545 & 6 & 0.1081 & -0.811 & -0.9660 & -1.03 & -0.51 & & & \\
\hline 3347.83 & 49492.711 & $1 / 2$ & 19631.205 & $3 / 2$ & 0.0705 & 14 & 0.046 & -1.152 & -1.3371 & -1.17 & -1.24 & & & \\
\hline 3353.12 & 49838.379 & $9 / 2$ & 20024.012 & $7 / 2$ & 0.0244 & 42 & 0.0203 & -1.613 & -1.6918 & -1.477 & -0.97 & & & \\
\hline 3358.50 & 49564.504 & $3 / 2$ & 19797.859 & $5 / 2$ & 0.2143 & 13 & 0.1399 & -0.669 & -0.8543 & -0.722 & -0.44 & & & \\
\hline 3368.05 & 49706.262 & $5 / 2$ & 20024.012 & $7 / 2$ & 0.6839 & 5 & 0.4469 & -0.165 & -0.3498 & -0.319 & 0.18 & & & \\
\hline 3382.68 & 49351.734 & $5 / 2$ & 19797.859 & $5 / 2$ & 0.1052 & 8 & 0.1567 & -0.978 & -0.8048 & -0.639 & -0.7 & & & -0.354 \\
\hline 3391.43 & 49005.848 & $3 / 2$ & 19528.23 & $1 / 2$ & 0.04 & 11 & 0.0342 & -1.398 & -1.4655 & -1.35 & & & & -0.906 \\
\hline 3403.26 & 54417.957 & $1 / 2$ & 25042.76 & $3 / 2$ & 0.067 & 16 & 0.0702 & -1.174 & -1.1537 & -1.054 & & & & -0.508 \\
\hline 3403.32 & 49005.848 & $3 / 2$ & 19631.205 & $3 / 2$ & 0.2168 & 7 & 0.2121 & -0.664 & -0.6735 & -0.536 & & & & \\
\hline 3408.77 & 49351.734 & $5 / 2$ & 20024.012 & $7 / 2$ & 0.3767 & 9 & 0.5267 & -0.424 & -0.2784 & -0.038 & -0.048 & & & -0.017 \\
\hline 3421.21 & 48749.277 & $1 / 2$ & 19528.23 & $1 / 2$ & 0.1905 & 7 & 0.1742 & -0.720 & -0.7589 & -0.611 & & & & -0.224 \\
\hline 3422.74 & 49005.848 & $3 / 2$ & 19797.859 & $5 / 2$ & 0.3776 & 6 & 0.3515 & -0.423 & -0.4541 & -0.266 & -0.15 & & & -0.039 \\
\hline 3433.31 & 48749.277 & $1 / 2$ & 19631.205 & $3 / 2$ & 0.1866 & 7 & 0.158 & -0.729 & -0.8014 & -0.624 & & & & -0.338 \\
\hline 3475.13 & 48398.871 & $3 / 2$ & 19631.205 & $3 / 2$ & 0.024 & 20 & 0.0105 & -1.620 & -1.9808 & -1.764 & & & & \\
\hline 3495.38 & 48398.871 & $3 / 2$ & 19797.859 & $5 / 2$ & 0.0197 & 22 & 0.0152 & -1.706 & -1.8187 & -1.539 & & & & \\
\hline 3511.83 & 48491.059 & $5 / 2$ & 20024.012 & $7 / 2$ & 0.0346 & 17 & 0.019 & -1.461 & -1.7205 & -1.452 & -1.46 & & & -1.074 \\
\hline 3585.30 & 49706.262 & $5 / 2$ & 21822.506 & $5 / 2$ & 0.1991 & 6 & 0.0315 & -0.701 & -1.5012 & -1.144 & & & & \\
\hline 3585.50 & 49706.262 & $5 / 2$ & 21824.141 & $3 / 2$ & 0.0412 & 10 & 0.0151 & -1.385 & -1.8203 & -1.517 & & & & \\
\hline 3631.47 & 49351.734 & $5 / 2$ & 21822.506 & $5 / 2$ & 0.0594 & 10 & 0.0364 & -1.226 & -1.4388 & -0.846 & & & & \\
\hline 3631.68 & 49351.734 & $5 / 2$ & 21824.141 & $3 / 2$ & 0.0208 & 16 & 0.0181 & -1.682 & -1.7428 & -1.253 & & & & \\
\hline 3677.68 & 49005.848 & $3 / 2$ & 21822.506 & $5 / 2$ & 0.0308 & 11 & 0.045 & -1.511 & -1.3470 & -1.266 & & & & \\
\hline 3677.84 & 49005.848 & $3 / 2$ & 21823.725 & $1 / 2$ & 0.0314 & 11 & 0.0212 & -1.503 & -1.6737 & -1.247 & & & & \\
\hline 3712.95 & 48749.277 & $1 / 2$ & 21824.141 & $3 / 2$ & 0.0469 & 9 & 0.0353 & -1.329 & -1.4521 & -1.243 & & & & \\
\hline
\end{tabular}


Table 5

Transition radial integral values in Cr II.

\begin{tabular}{llll}
\hline Transition & HFR & HFR+CPOL & OSP \\
\hline $3 \mathrm{~d}^{4} 4 \mathrm{~s}-3 \mathrm{~d}^{4} 4 \mathrm{p}$ & 3.184 & 2.969 & $2.932 \pm 0.003$ \\
$3 \mathrm{~d}^{5}-3 \mathrm{~d}^{4} 4 \mathrm{p}$ & 1.567 & 1.503 & $1.413 \pm 0.008$ \\
$3 \mathrm{~d}^{4} 4 \mathrm{~d}-3 \mathrm{~d}^{4} 4 \mathrm{p}$ & 5.097 & 4.944 & $5.11 \pm 0.09$ \\
\hline
\end{tabular}


Table 6

Semi-empirical radiative data in $\mathrm{Cr}$ II.

\begin{tabular}{|c|c|c|c|c|c|c|c|c|c|c|}
\hline $\begin{array}{l}\text { Wavelength } \\
(\AA)\end{array}$ & $\begin{array}{l}E \text { (Lower) } \\
\left(\mathrm{cm}^{-1}\right)\end{array}$ & Parity (Lower) & $J$ (Lower) & $\begin{array}{l}E \text { (Upper) } \\
\left(\mathrm{cm}^{-1}\right)\end{array}$ & Parity (Upper) & $J$ (Upper) & $\log (g f)(\mathrm{HFR}+\mathrm{CPOL})$ & $\log (g f)$ (Kurucz) & $\begin{array}{l}g A(\mathrm{HFR}+\mathrm{CPOL}) \\
\left(\mathrm{s}^{-1}\right)\end{array}$ & $B F(\mathrm{HFR}+\mathrm{CPOL})$ \\
\hline 1825.335 & 0 & (e) & 2.5 & 54784 & (o) & 3.5 & -3.28 & -2.944 & $1.07 \mathrm{E}+06$ & $5.75 \mathrm{E}-04$ \\
\hline 1830.644 & 0 & (e) & 2.5 & 54626 & (o) & 2.5 & -3.93 & -3.602 & $2.38 \mathrm{E}+05$ & $1.71 \mathrm{E}-04$ \\
\hline 2011.169 & 0 & (e) & 2.5 & 49706 & (o) & 2.5 & -2.73 & -3.960 & $3.14 \mathrm{E}+06$ & $2.36 \mathrm{E}-03$ \\
\hline 2016.922 & 0 & (e) & 2.5 & 49565 & (o) & 1.5 & -3.33 & -4.848 & $7.79 \mathrm{E}+05$ & $8.18 \mathrm{E}-04$ \\
\hline 2025.619 & 0 & (e) & 2.5 & 49352 & (o) & 2.5 & -2.42 & -1.549 & $6.20 \mathrm{E}+06$ & $4.75 \mathrm{E}-03$ \\
\hline 2039.918 & 0 & (e) & 2.5 & 49006 & (o) & 1.5 & -2.43 & -1.577 & $6.05 \mathrm{E}+06$ & $7.11 \mathrm{E}-03$ \\
\hline 2055.599 & 0 & (e) & 2.5 & 48632 & (o) & 3.5 & -0.03 & 0.025 & $1.48 \mathrm{E}+09$ & $4.44 \mathrm{E}-01$ \\
\hline 2061.577 & 0 & (e) & 2.5 & 48491 & (o) & 2.5 & -0.16 & -0.114 & $1.09 \mathrm{E}+09$ & $4.18 \mathrm{E}-01$ \\
\hline 2065.504 & 0 & (e) & 2.5 & 48399 & (o) & 1.5 & -0.34 & -0.297 & $7.20 \mathrm{E}+08$ & $4.32 \mathrm{E}-01$ \\
\hline 2344.680 & 12148 & (e) & 2.5 & 54784 & (o) & 3.5 & -3.85 & -3.463 & $1.72 \mathrm{E}+05$ & $9.25 \mathrm{E}-05$ \\
\hline 2347.082 & 12033 & (e) & 1.5 & 54626 & (o) & 2.5 & -3.65 & -3.296 & $2.70 \mathrm{E}+05$ & $1.94 \mathrm{E}-04$ \\
\hline 2350.134 & 11962 & (e) & 0.5 & 54499 & (o) & 1.5 & -3.74 & -3.430 & $2.19 \mathrm{E}+05$ & $2.35 \mathrm{E}-04$ \\
\hline 2353.449 & 12148 & (e) & 2.5 & 54626 & (o) & 2.5 & -3.12 & -2.985 & $9.20 \mathrm{E}+05$ & $6.59 \mathrm{E}-04$ \\
\hline 2354.052 & 12033 & (e) & 1.5 & 54499 & (o) & 1.5 & -3.22 & -3.044 & $7.25 \mathrm{E}+05$ & $7.79 \mathrm{E}-04$ \\
\hline 2354.648 & 11962 & (e) & 0.5 & 54418 & (o) & 0.5 & -3.41 & -3.203 & $4.67 \mathrm{E}+05$ & $1.00 \mathrm{E}-03$ \\
\hline 2362.128 & 12304 & (e) & 3.5 & 54626 & (o) & 2.5 & -3.76 & -3.274 & $2.10 \mathrm{E}+05$ & $1.51 \mathrm{E}-04$ \\
\hline 2521.879 & 12148 & (e) & 2.5 & 51789 & (o) & 3.5 & -3.43 & -4.800 & $3.85 \mathrm{E}+05$ & $1.97 \mathrm{E}-04$ \\
\hline 2527.585 & 12033 & (e) & 1.5 & 51584 & (o) & 1.5 & -3.54 & -3.240 & $3.00 \mathrm{E}+05$ & $3.15 \mathrm{E}-04$ \\
\hline 2529.499 & 12148 & (e) & 2.5 & 51669 & (o) & 2.5 & -2.68 & -2.477 & $2.17 E+06$ & $1.48 \mathrm{E}-03$ \\
\hline 2534.971 & 12148 & (e) & 2.5 & 51584 & (o) & 1.5 & -3.43 & -3.329 & $3.81 \mathrm{E}+05$ & $4.00 \mathrm{E}-04$ \\
\hline 2539.527 & 12304 & (e) & 3.5 & 51669 & (o) & 2.5 & -2.82 & -2.764 & $1.56 \mathrm{E}+06$ & $1.07 \mathrm{E}-03$ \\
\hline 2653.581 & 12033 & (e) & 1.5 & 49706 & (o) & 2.5 & -0.46 & -0.650 & $3.28 \mathrm{E}+08$ & $2.46 \mathrm{E}-01$ \\
\hline 2658.588 & 11962 & (e) & 0.5 & 49565 & (o) & 1.5 & -0.50 & -0.610 & $2.98 \mathrm{E}+08$ & $3.13 \mathrm{E}-01$ \\
\hline 2661.722 & 12148 & (e) & 2.5 & 49706 & (o) & 2.5 & -0.91 & -0.755 & $1.16 \mathrm{E}+08$ & $8.70 \mathrm{E}-02$ \\
\hline 2663.604 & 12033 & (e) & 1.5 & 49565 & (o) & 1.5 & -2.29 & -2.181 & $4.87 \mathrm{E}+06$ & $5.11 \mathrm{E}-03$ \\
\hline 2663.674 & 11962 & (e) & 0.5 & 49493 & (o) & 0.5 & -0.98 & -0.973 & $9.80 \mathrm{E}+07$ & $2.11 \mathrm{E}-01$ \\
\hline 2666.014 & 12148 & (e) & 2.5 & 49646 & (o) & 3.5 & -0.12 & -0.300 & $7.23 \mathrm{E}+08$ & $3.43 \mathrm{E}-01$ \\
\hline 2668.709 & 12033 & (e) & 1.5 & 49493 & (o) & 0.5 & -0.44 & -0.520 & $3.40 \mathrm{E}+08$ & $7.31 \mathrm{E}-01$ \\
\hline 2671.807 & 12148 & (e) & 2.5 & 49565 & (o) & 1.5 & -0.26 & -0.370 & $5.15 \mathrm{E}+08$ & $5.41 \mathrm{E}-01$ \\
\hline 2672.828 & 12304 & (e) & 3.5 & 49706 & (o) & 2.5 & -0.33 & -0.450 & $4.42 \mathrm{E}+08$ & $3.32 \mathrm{E}-01$ \\
\hline 2678.791 & 12033 & (e) & 1.5 & 49352 & (o) & 2.5 & -0.43 & -0.475 & $3.50 \mathrm{E}+08$ & $2.68 \mathrm{E}-01$ \\
\hline 2687.088 & 12148 & (e) & 2.5 & 49352 & (o) & 2.5 & -0.71 & -0.674 & $1.82 \mathrm{E}+08$ & $1.40 \mathrm{E}-01$ \\
\hline 2698.407 & 12304 & (e) & 3.5 & 49352 & (o) & 2.5 & -0.58 & -1.077 & $2.45 \mathrm{E}+08$ & $1.88 \mathrm{E}-01$ \\
\hline 2698.684 & 11962 & (e) & 0.5 & 49006 & (o) & 1.5 & -0.79 & -0.656 & $1.48 \mathrm{E}+08$ & $1.74 \mathrm{E}-01$ \\
\hline 2703.852 & 12033 & (e) & 1.5 & 49006 & (o) & 1.5 & -1.78 & -1.327 & $1.53 \mathrm{E}+07$ & $1.80 \mathrm{E}-02$ \\
\hline 2712.306 & 12148 & (e) & 2.5 & 49006 & (o) & 1.5 & -0.86 & -1.167 & $1.26 \mathrm{E}+08$ & $1.48 \mathrm{E}-01$ \\
\hline 2717.507 & 11962 & (e) & 0.5 & 48749 & (o) & 0.5 & -1.58 & -1.543 & $2.40 \mathrm{E}+07$ & $6.00 \mathrm{E}-02$ \\
\hline 2722.747 & 12033 & (e) & 1.5 & 48749 & (o) & 0.5 & -1.02 & -1.014 & $8.54 \mathrm{E}+07$ & $2.14 \mathrm{E}-01$ \\
\hline 2740.095 & 12148 & (e) & 2.5 & 48632 & (o) & 3.5 & -1.05 & -1.000 & $7.87 \mathrm{E}+07$ & $2.36 \mathrm{E}-02$ \\
\hline 2742.032 & 12033 & (e) & 1.5 & 48491 & (o) & 2.5 & -0.67 & -0.817 & $1.88 \mathrm{E}+08$ & $7.21 \mathrm{E}-02$ \\
\hline 2743.642 & 11962 & (e) & 0.5 & 48399 & (o) & 1.5 & -0.45 & -0.545 & $3.12 \mathrm{E}+08$ & $1.87 \mathrm{E}-01$ \\
\hline 2748.984 & 12033 & (e) & 1.5 & 48399 & (o) & 1.5 & -0.29 & -0.305 & $4.47 \mathrm{E}+08$ & $2.68 \mathrm{E}-01$ \\
\hline 2750.727 & 12148 & (e) & 2.5 & 48491 & (o) & 2.5 & -0.18 & -0.226 & $5.78 \mathrm{E}+08$ & $2.22 \mathrm{E}-01$ \\
\hline 2757.722 & 12148 & (e) & 2.5 & 48399 & (o) & 1.5 & -0.44 & -0.349 & $3.14 \mathrm{E}+08$ & $1.88 \mathrm{E}-01$ \\
\hline 2762.589 & 12304 & (e) & 3.5 & 48491 & (o) & 2.5 & -0.02 & 0.048 & $8.36 \mathrm{E}+08$ & $3.20 \mathrm{E}-01$ \\
\hline 2849.834 & 12148 & (e) & 2.5 & 47227 & (o) & 3.5 & 0.18 & -0.050 & $1.26 \mathrm{E}+09$ & $6.46 \mathrm{E}-01$ \\
\hline 2855.673 & 12033 & (e) & 1.5 & 47040 & (o) & 2.5 & -0.07 & -0.069 & $7.01 \mathrm{E}+08$ & $4.91 \mathrm{E}-01$ \\
\hline 2856.762 & 19631 & (e) & 1.5 & 54626 & (o) & 2.5 & -0.65 & -0.500 & $1.82 \mathrm{E}+08$ & $1.30 \mathrm{E}-01$ \\
\hline 2857.398 & 19798 & (e) & 2.5 & 54784 & (o) & 3.5 & -0.75 & -0.560 & $1.47 \mathrm{E}+08$ & $7.90 \mathrm{E}-02$ \\
\hline 2858.651 & 19528 & (e) & 0.5 & 54499 & (o) & 1.5 & -0.79 & -0.726 & $1.34 \mathrm{E}+08$ & $1.44 \mathrm{E}-01$ \\
\hline 2860.931 & 11962 & (e) & 0.5 & 46905 & (o) & 1.5 & -0.43 & -0.470 & $3.02 \mathrm{E}+08$ & $3.17 \mathrm{E}-01$ \\
\hline 2865.104 & 12148 & (e) & 2.5 & 47040 & (o) & 2.5 & -0.08 & -0.057 & $6.71 \mathrm{E}+08$ & $4.70 \mathrm{E}-01$ \\
\hline 2865.332 & 19528 & (e) & 0.5 & 54418 & (o) & 0.5 & -0.76 & -0.688 & $1.42 \mathrm{E}+08$ & $3.05 \mathrm{E}-01$ \\
\hline
\end{tabular}


Table 6 (continued)

\begin{tabular}{|c|c|c|c|c|c|c|c|c|c|c|}
\hline $\begin{array}{l}\text { Wavelength } \\
(\AA \AA)\end{array}$ & $\begin{array}{l}E(\text { Lower }) \\
\left(\mathrm{cm}^{-1}\right)\end{array}$ & Parity (Lower) & $J$ (Lower) & $\begin{array}{l}E \text { (Upper) } \\
\left(\mathrm{cm}^{-1}\right)\end{array}$ & Parity (Upper) & $J$ (Upper) & $\log (g f)(\mathrm{HFR}+\mathrm{CPOL})$ & $\log (g f)$ (Kurucz) & $\begin{array}{l}g A(\mathrm{HFR}+\mathrm{CPOL}) \\
\left(\mathrm{s}^{-1}\right)\end{array}$ & $B F(\mathrm{HFR}+\mathrm{CPOL})$ \\
\hline 2866.740 & 12033 & (e) & 1.5 & 46905 & (o) & 1.5 & -0.17 & -0.230 & $5.49 \mathrm{E}+08$ & $5.76 \mathrm{E}-01$ \\
\hline 2867.094 & 19631 & (e) & 1.5 & 54499 & (o) & 1.5 & -0.55 & -0.270 & $2.29 \mathrm{E}+08$ & $2.46 \mathrm{E}-01$ \\
\hline 2867.647 & 11962 & (e) & 0.5 & 46823 & (o) & 0.5 & -0.34 & -0.570 & $3.75 \mathrm{E}+08$ & $8.06 \mathrm{E}-01$ \\
\hline 2870.432 & 19798 & (e) & 2.5 & 54626 & (o) & 2.5 & -0.21 & -0.020 & $4.99 \mathrm{E}+08$ & $3.58 \mathrm{E}-01$ \\
\hline 2873.483 & 12033 & (e) & 1.5 & 46823 & (o) & 0.5 & -0.88 & -0.853 & $1.06 \mathrm{E}+08$ & $2.28 \mathrm{E}-01$ \\
\hline 2873.814 & 19631 & (e) & 1.5 & 54418 & (o) & 0.5 & -0.73 & -0.660 & $1.50 \mathrm{E}+08$ & $3.23 \mathrm{E}-01$ \\
\hline 2876.244 & 12148 & (e) & 2.5 & 46905 & (o) & 1.5 & -0.85 & -0.804 & $1.14 \mathrm{E}+08$ & $1.20 \mathrm{E}-01$ \\
\hline 2877.975 & 12304 & (e) & 3.5 & 47040 & (o) & 2.5 & -1.00 & -0.931 & $8.11 \mathrm{E}+07$ & $5.68 \mathrm{E}-02$ \\
\hline 2880.864 & 19798 & (e) & 2.5 & 54499 & (o) & 1.5 & -0.57 & -0.410 & $2.18 \mathrm{E}+08$ & $2.34 \mathrm{E}-01$ \\
\hline 2889.194 & 20024 & (e) & 3.5 & 54626 & (o) & 2.5 & -0.63 & -0.554 & $1.88 \mathrm{E}+08$ & $1.35 \mathrm{E}-01$ \\
\hline 3032.919 & 21823 & (e) & 2.5 & 54784 & (o) & 3.5 & -1.12 & -1.031 & $5.46 \mathrm{E}+07$ & $2.93 \mathrm{E}-02$ \\
\hline 3047.607 & 21823 & (e) & 2.5 & 54626 & (o) & 2.5 & -1.21 & -1.234 & $4.41 \mathrm{E}+07$ & $3.16 \mathrm{E}-02$ \\
\hline 3047.759 & 21824 & (e) & 1.5 & 54626 & (o) & 2.5 & -1.43 & -1.326 & $2.68 \mathrm{E}+07$ & $1.92 \mathrm{E}-02$ \\
\hline 3059.368 & 21823 & (e) & 2.5 & 54499 & (o) & 1.5 & -1.88 & -1.941 & $9.39 \mathrm{E}+06$ & $1.01 \mathrm{E}-02$ \\
\hline 3059.482 & 21824 & (e) & 0.5 & 54499 & (o) & 1.5 & -1.76 & -1.675 & $1.23 \mathrm{E}+07$ & $1.32 \mathrm{E}-02$ \\
\hline 3059.521 & 21824 & (e) & 1.5 & 54499 & (o) & 1.5 & -1.30 & -1.287 & $3.58 \mathrm{E}+07$ & $3.85 \mathrm{E}-02$ \\
\hline 3067.136 & 21824 & (e) & 0.5 & 54418 & (o) & 0.5 & -1.51 & -1.473 & $2.20 \mathrm{E}+07$ & $4.73 \mathrm{E}-02$ \\
\hline 3067.175 & 21824 & (e) & 1.5 & 54418 & (o) & 0.5 & -1.91 & -1.927 & $8.77 \mathrm{E}+06$ & $1.89 \mathrm{E}-02$ \\
\hline 3118.649 & 19528 & (e) & 0.5 & 51584 & (o) & 1.5 & -0.11 & 0.000 & $5.25 \mathrm{E}+08$ & $5.51 \mathrm{E}-01$ \\
\hline 3120.369 & 19631 & (e) & 1.5 & 51669 & (o) & 2.5 & 0.09 & 0.120 & $8.39 \mathrm{E}+08$ & $5.73 \mathrm{E}-01$ \\
\hline 3124.977 & 19798 & (e) & 2.5 & 51789 & (o) & 3.5 & 0.27 & -0.018 & $1.26 \mathrm{E}+09$ & $6.46 \mathrm{E}-01$ \\
\hline 3128.700 & 19631 & (e) & 1.5 & 51584 & (o) & 1.5 & -0.54 & -0.320 & $1.96 \mathrm{E}+08$ & $2.06 \mathrm{E}-01$ \\
\hline 3136.686 & 19798 & (e) & 2.5 & 51669 & (o) & 2.5 & -0.44 & -0.250 & $2.44 \mathrm{E}+08$ & $1.67 \mathrm{E}-01$ \\
\hline 3145.104 & 19798 & (e) & 2.5 & 51584 & (o) & 1.5 & -1.75 & -1.733 & $1.20 \mathrm{E}+07$ & $1.26 \mathrm{E}-02$ \\
\hline 3159.103 & 20024 & (e) & 3.5 & 51669 & (o) & 2.5 & -1.94 & -1.950 & $7.58 \mathrm{E}+06$ & $5.18 \mathrm{E}-03$ \\
\hline 3196.339 & 20512 & (e) & 2.5 & 51789 & (o) & 3.5 & -2.88 & -2.827 & $8.44 \mathrm{E}+05$ & $4.33 \mathrm{E}-04$ \\
\hline 3208.589 & 20512 & (e) & 2.5 & 51669 & (o) & 2.5 & -1.33 & -1.307 & $3.03 \mathrm{E}+07$ & $2.07 \mathrm{E}-02$ \\
\hline 3209.179 & 20518 & (e) & 3.5 & 51669 & (o) & 2.5 & -0.40 & -0.200 & $2.58 \mathrm{E}+08$ & $1.76 \mathrm{E}-01$ \\
\hline 3217.398 & 20512 & (e) & 2.5 & 51584 & (o) & 1.5 & -0.52 & -0.320 & $1.91 \mathrm{E}+08$ & $2.01 \mathrm{E}-01$ \\
\hline 3324.058 & 19631 & (e) & 1.5 & 49706 & (o) & 2.5 & -1.60 & -1.691 & $1.53 \mathrm{E}+07$ & $1.15 \mathrm{E}-02$ \\
\hline 3328.350 & 19528 & (e) & 0.5 & 49565 & (o) & 1.5 & -1.72 & -1.752 & $1.15 \mathrm{E}+07$ & $1.21 \mathrm{E}-02$ \\
\hline 3336.121 & 21823 & (e) & 2.5 & 51789 & (o) & 3.5 & -2.15 & -2.695 & $4.20 \mathrm{E}+06$ & $2.15 \mathrm{E}-03$ \\
\hline 3336.325 & 19528 & (e) & 0.5 & 49493 & (o) & 0.5 & -1.19 & -0.850 & $3.89 \mathrm{E}+07$ & $8.36 \mathrm{E}-02$ \\
\hline 3339.801 & 19631 & (e) & 1.5 & 49565 & (o) & 1.5 & -1.01 & -0.480 & $5.82 \mathrm{E}+07$ & $6.11 \mathrm{E}-02$ \\
\hline 3342.581 & 19798 & (e) & 2.5 & 49706 & (o) & 2.5 & -0.87 & -0.410 & $8.00 \mathrm{E}+07$ & $6.00 \mathrm{E}-02$ \\
\hline 3347.831 & 19631 & (e) & 1.5 & 49493 & (o) & 0.5 & -1.19 & -0.760 & $3.87 \mathrm{E}+07$ & $8.32 \mathrm{E}-02$ \\
\hline 3349.351 & 19798 & (e) & 2.5 & 49646 & (o) & 3.5 & -2.16 & -2.128 & $4.15 \mathrm{E}+06$ & $1.97 \mathrm{E}-03$ \\
\hline 3349.469 & 21823 & (e) & 2.5 & 51669 & (o) & 2.5 & -2.91 & -3.520 & $7.29 \mathrm{E}+05$ & $4.98 \mathrm{E}-04$ \\
\hline 3349.652 & 21824 & (e) & 1.5 & 51669 & (o) & 2.5 & -2.55 & -3.123 & $1.66 \mathrm{E}+06$ & $1.13 \mathrm{E}-03$ \\
\hline 3358.500 & 19798 & (e) & 2.5 & 49565 & (o) & 1.5 & -0.69 & -0.130 & $1.20 \mathrm{E}+08$ & $1.26 \mathrm{E}-01$ \\
\hline 3359.207 & 21824 & (e) & 0.5 & 51584 & (o) & 1.5 & -3.32 & -4.773 & $2.79 \mathrm{E}+05$ & $2.93 \mathrm{E}-04$ \\
\hline 3359.254 & 21824 & (e) & 1.5 & 51584 & (o) & 1.5 & -3.21 & -3.925 & $3.56 \mathrm{E}+05$ & $3.74 \mathrm{E}-04$ \\
\hline 3361.765 & 25047 & (e) & 2.5 & 54784 & (o) & 3.5 & -0.84 & -0.926 & $8.48 \mathrm{E}+07$ & $4.56 \mathrm{E}-02$ \\
\hline 3363.711 & 19631 & (e) & 1.5 & 49352 & (o) & 2.5 & -1.88 & -1.695 & $7.88 \mathrm{E}+06$ & $6.04 \mathrm{E}-03$ \\
\hline 3368.049 & 20024 & (e) & 3.5 & 49706 & (o) & 2.5 & -0.18 & 0.150 & $3.93 \mathrm{E}+08$ & $2.95 \mathrm{E}-01$ \\
\hline 3378.330 & 25034 & (e) & 3.5 & 54626 & (o) & 2.5 & -0.81 & -0.380 & $9.06 \mathrm{E}+07$ & $6.49 \mathrm{E}-02$ \\
\hline 3379.368 & 25043 & (e) & 1.5 & 54626 & (o) & 2.5 & -0.79 & -0.310 & $9.57 \mathrm{E}+07$ & $6.86 \mathrm{E}-02$ \\
\hline 3379.820 & 25047 & (e) & 2.5 & 54626 & (o) & 2.5 & -0.43 & -0.548 & $2.18 \mathrm{E}+08$ & $1.56 \mathrm{E}-01$ \\
\hline 3382.680 & 19798 & (e) & 2.5 & 49352 & (o) & 2.5 & -0.75 & -0.330 & $1.04 \mathrm{E}+08$ & $7.97 \mathrm{E}-02$ \\
\hline 3391.431 & 19528 & (e) & 0.5 & 49006 & (o) & 1.5 & -1.37 & -0.880 & $2.49 \mathrm{E}+07$ & $2.93 \mathrm{E}-02$ \\
\hline 3392.981 & 25035 & (e) & 0.5 & 54499 & (o) & 1.5 & -0.95 & -0.500 & $6.49 \mathrm{E}+07$ & $6.98 \mathrm{E}-02$ \\
\hline 3393.836 & 25043 & (e) & 1.5 & 54499 & (o) & 1.5 & -0.78 & -0.340 & $9.61 \mathrm{E}+07$ & $1.03 \mathrm{E}-01$ \\
\hline 3394.291 & 25047 & (e) & 2.5 & 54499 & (o) & 1.5 & -0.77 & -0.290 & $9.90 \mathrm{E}+07$ & $1.06 \mathrm{E}-01$ \\
\hline 3402.397 & 25035 & (e) & 0.5 & 54418 & (o) & 0.5 & -0.97 & -0.560 & $6.14 \mathrm{E}+07$ & $1.32 \mathrm{E}-01$ \\
\hline
\end{tabular}


Table 6 (continued)

\begin{tabular}{|c|c|c|c|c|c|c|c|c|c|c|}
\hline $\begin{array}{l}\text { Wavelength } \\
(\AA)\end{array}$ & $\begin{array}{l}E \text { (Lower) } \\
\left(\mathrm{cm}^{-1}\right)\end{array}$ & Parity (Lower) & $J$ (Lower) & $\begin{array}{l}E \text { (Upper) } \\
\left(\mathrm{cm}^{-1}\right)\end{array}$ & Parity (Upper) & $J$ (Upper) & $\log (g f)(\mathrm{HFR}+\mathrm{CPOL})$ & $\log (g f)$ (Kurucz) & $\begin{array}{l}g A(\mathrm{HFR}+\mathrm{CPOL}) \\
\left(\mathrm{s}^{-1}\right)\end{array}$ & $B F(\mathrm{HFR}+\mathrm{CPOL})$ \\
\hline 3403.256 & 25043 & (e) & 1.5 & 54418 & (o) & 0.5 & -0.94 & -1.054 & $6.60 \mathrm{E}+07$ & $1.42 \mathrm{E}-01$ \\
\hline 3403.320 & 19631 & (e) & 1.5 & 49006 & (o) & 1.5 & -0.54 & -0.536 & $1.65 \mathrm{E}+08$ & $1.94 \mathrm{E}-01$ \\
\hline 3408.765 & 20024 & (e) & 3.5 & 49352 & (o) & 2.5 & -0.14 & 0.000 & $4.18 \mathrm{E}+08$ & $3.20 \mathrm{E}-01$ \\
\hline 3421.209 & 19528 & (e) & 0.5 & 48749 & (o) & 0.5 & -0.62 & -0.220 & $1.39 \mathrm{E}+08$ & $3.48 \mathrm{E}-01$ \\
\hline 3422.739 & 19798 & (e) & 2.5 & 49006 & (o) & 1.5 & -0.26 & -0.010 & $3.12 \mathrm{E}+08$ & $3.67 \mathrm{E}-01$ \\
\hline 3425.028 & 20518 & (e) & 3.5 & 49706 & (o) & 2.5 & -3.79 & -3.520 & $9.13 \mathrm{E}+04$ & $6.85 \mathrm{E}-05$ \\
\hline 3433.309 & 19631 & (e) & 1.5 & 48749 & (o) & 0.5 & -0.62 & -0.340 & $1.36 \mathrm{E}+08$ & $3.40 \mathrm{E}-01$ \\
\hline 3462.734 & 19528 & (e) & 0.5 & 48399 & (o) & 1.5 & -3.15 & -2.541 & $3.87 \mathrm{E}+05$ & $2.32 \mathrm{E}-04$ \\
\hline 3464.029 & 19631 & (e) & 1.5 & 48491 & (o) & 2.5 & -3.19 & -2.769 & $3.54 \mathrm{E}+05$ & $1.36 \mathrm{E}-04$ \\
\hline 3467.111 & 19798 & (e) & 2.5 & 48632 & (o) & 3.5 & -3.72 & -3.655 & $1.04 \mathrm{E}+05$ & $3.12 \mathrm{E}-05$ \\
\hline 3467.142 & 20518 & (e) & 3.5 & 49352 & (o) & 2.5 & -3.54 & -3.791 & $1.60 \mathrm{E}+05$ & $1.23 \mathrm{E}-04$ \\
\hline 3475.130 & 19631 & (e) & 1.5 & 48399 & (o) & 1.5 & -2.39 & -1.764 & $2.20 \mathrm{E}+06$ & $1.32 \mathrm{E}-03$ \\
\hline 3484.149 & 19798 & (e) & 2.5 & 48491 & (o) & 2.5 & -2.40 & -1.903 & $2.16 \mathrm{E}+06$ & $8.28 \mathrm{E}-04$ \\
\hline 3495.379 & 19798 & (e) & 2.5 & 48399 & (o) & 1.5 & -2.17 & -1.824 & $3.65 \mathrm{E}+06$ & $2.19 \mathrm{E}-03$ \\
\hline 3511.829 & 20024 & (e) & 3.5 & 48491 & (o) & 2.5 & -1.95 & -1.060 & $5.99 \mathrm{E}+06$ & $2.30 \mathrm{E}-03$ \\
\hline 3585.294 & 21823 & (e) & 2.5 & 49706 & (o) & 2.5 & -0.97 & -1.144 & $5.57 \mathrm{E}+07$ & $4.18 \mathrm{E}-02$ \\
\hline 3585.504 & 21824 & (e) & 1.5 & 49706 & (o) & 2.5 & -1.36 & -1.517 & $2.26 \mathrm{E}+07$ & $1.70 \mathrm{E}-02$ \\
\hline 3603.615 & 21823 & (e) & 2.5 & 49565 & (o) & 1.5 & -1.79 & -1.722 & $8.36 \mathrm{E}+06$ & $8.78 \mathrm{E}-03$ \\
\hline 3603.773 & 21824 & (e) & 0.5 & 49565 & (o) & 1.5 & -1.66 & -1.693 & $1.13 \mathrm{E}+07$ & $1.19 \mathrm{E}-02$ \\
\hline 3603.827 & 21824 & (e) & 1.5 & 49565 & (o) & 1.5 & -2.04 & -2.148 & $4.64 \mathrm{E}+06$ & $4.87 \mathrm{E}-03$ \\
\hline 3613.124 & 21824 & (e) & 0.5 & 49493 & (o) & 0.5 & -2.56 & -2.480 & $1.40 \mathrm{E}+06$ & $3.01 \mathrm{E}-03$ \\
\hline 3613.179 & 21824 & (e) & 1.5 & 49493 & (o) & 0.5 & -1.85 & -1.779 & $7.24 \mathrm{E}+06$ & $1.56 \mathrm{E}-02$ \\
\hline 3631.467 & 21823 & (e) & 2.5 & 49352 & (o) & 2.5 & -0.93 & -0.846 & $6.02 \mathrm{E}+07$ & $4.62 \mathrm{E}-02$ \\
\hline 3631.683 & 21824 & (e) & 1.5 & 49352 & (o) & 2.5 & -1.35 & -1.253 & $2.27 \mathrm{E}+07$ & $1.74 \mathrm{E}-02$ \\
\hline 3644.690 & 19798 & (e) & 2.5 & 47227 & (o) & 3.5 & -2.74 & -2.379 & $9.08 \mathrm{E}+05$ & $4.65 \mathrm{E}-04$ \\
\hline 3647.388 & 19631 & (e) & 1.5 & 47040 & (o) & 2.5 & -2.92 & -2.600 & $5.98 \mathrm{E}+05$ & $4.19 \mathrm{E}-04$ \\
\hline 3651.673 & 19528 & (e) & 0.5 & 46905 & (o) & 1.5 & -3.28 & -2.993 & $2.63 \mathrm{E}+05$ & $2.76 \mathrm{E}-04$ \\
\hline 3662.621 & 19528 & (e) & 0.5 & 46823 & (o) & 0.5 & -3.95 & -3.788 & $5.55 \mathrm{E}+04$ & $1.19 \mathrm{E}-04$ \\
\hline 3677.676 & 21823 & (e) & 2.5 & 49006 & (o) & 1.5 & -1.38 & -1.420 & $2.07 \mathrm{E}+07$ & $2.43 \mathrm{E}-02$ \\
\hline 3677.841 & 21824 & (e) & 0.5 & 49006 & (o) & 1.5 & -1.25 & -1.390 & $2.79 \mathrm{E}+07$ & $3.28 \mathrm{E}-02$ \\
\hline 3677.898 & 21824 & (e) & 1.5 & 49006 & (o) & 1.5 & -1.61 & -1.854 & $1.22 \mathrm{E}+07$ & $1.43 \mathrm{E}-02$ \\
\hline 3712.887 & 21824 & (e) & 0.5 & 48749 & (o) & 0.5 & -2.03 & -1.961 & $4.48 \mathrm{E}+06$ & $1.12 \mathrm{E}-02$ \\
\hline 3712.945 & 21824 & (e) & 1.5 & 48749 & (o) & 0.5 & -1.31 & -1.201 & $2.37 \mathrm{E}+07$ & $5.93 \mathrm{E}-02$ \\
\hline 3738.359 & 25047 & (e) & 2.5 & 51789 & (o) & 3.5 & -1.25 & -1.975 & $2.66 \mathrm{E}+07$ & $1.36 \mathrm{E}-02$ \\
\hline 3748.669 & 21823 & (e) & 2.5 & 48491 & (o) & 2.5 & -2.59 & -2.194 & $1.19 \mathrm{E}+06$ & $4.56 \mathrm{E}-04$ \\
\hline 3748.899 & 21824 & (e) & 1.5 & 48491 & (o) & 2.5 & -3.16 & -2.680 & $3.25 \mathrm{E}+05$ & $1.25 \mathrm{E}-04$ \\
\hline 3754.569 & 25043 & (e) & 1.5 & 51669 & (o) & 2.5 & -1.46 & -2.188 & $1.61 \mathrm{E}+07$ & $1.10 \mathrm{E}-02$ \\
\hline 3755.127 & 25047 & (e) & 2.5 & 51669 & (o) & 2.5 & -2.31 & -4.233 & $2.27 \mathrm{E}+06$ & $1.55 \mathrm{E}-03$ \\
\hline 3761.672 & 21823 & (e) & 2.5 & 48399 & (o) & 1.5 & -3.14 & -2.514 & $3.39 \mathrm{E}+05$ & $2.03 \mathrm{E}-04$ \\
\hline 3761.845 & 21824 & (e) & 0.5 & 48399 & (o) & 1.5 & -3.14 & -2.511 & $3.37 \mathrm{E}+05$ & $2.02 \mathrm{E}-04$ \\
\hline 3761.904 & 21824 & (e) & 1.5 & 48399 & (o) & 1.5 & -3.45 & -2.892 & $1.63 \mathrm{E}+05$ & $9.78 \mathrm{E}-05$ \\
\hline 3765.585 & 25035 & (e) & 0.5 & 51584 & (o) & 1.5 & -1.72 & -2.499 & $8.88 \mathrm{E}+06$ & $9.32 \mathrm{E}-03$ \\
\hline 3766.637 & 25043 & (e) & 1.5 & 51584 & (o) & 1.5 & -2.34 & -3.620 & $2.14 \mathrm{E}+06$ & $2.25 \mathrm{E}-03$ \\
\hline 4054.079 & 25047 & (e) & 2.5 & 49706 & (o) & 2.5 & -3.31 & -2.475 & $1.97 \mathrm{E}+05$ & $1.48 \mathrm{E}-04$ \\
\hline 4064.043 & 25047 & (e) & 2.5 & 49646 & (o) & 3.5 & -3.53 & -4.370 & $1.19 \mathrm{E}+05$ & $5.65 \mathrm{E}-05$ \\
\hline 4072.556 & 29952 & (e) & 0.5 & 54499 & (o) & 1.5 & -2.40 & -2.407 & $1.60 \mathrm{E}+06$ & $1.72 \mathrm{E}-03$ \\
\hline 4086.128 & 29952 & (e) & 0.5 & 54418 & (o) & 0.5 & -2.44 & -2.422 & $1.46 \mathrm{E}+06$ & $3.14 \mathrm{E}-03$ \\
\hline 4110.982 & 30307 & (e) & 1.5 & 54626 & (o) & 2.5 & -2.02 & -2.023 & $3.78 \mathrm{E}+06$ & $2.71 \mathrm{E}-03$ \\
\hline 4112.547 & 25043 & (e) & 1.5 & 49352 & (o) & 2.5 & -3.66 & -3.019 & $8.67 \mathrm{E}+04$ & $6.65 \mathrm{E}-05$ \\
\hline 4113.216 & 25047 & (e) & 2.5 & 49352 & (o) & 2.5 & -3.56 & -2.274 & $1.09 \mathrm{E}+05$ & $8.36 \mathrm{E}-05$ \\
\hline 4132.411 & 30307 & (e) & 1.5 & 54499 & (o) & 1.5 & -2.38 & -2.345 & $1.63 \mathrm{E}+06$ & $1.75 \mathrm{E}-03$ \\
\hline 4146.386 & 30307 & (e) & 1.5 & 54418 & (o) & 0.5 & -3.18 & -3.149 & $2.59 \mathrm{E}+05$ & $5.57 \mathrm{E}-04$ \\
\hline 4179.421 & 30864 & (e) & 2.5 & 54784 & (o) & 3.5 & -1.77 & -1.773 & $6.47 \mathrm{E}+06$ & $3.48 \mathrm{E}-03$ \\
\hline
\end{tabular}




E(Lower)

E(Upper)

Parity (Upper)

$J$ (Upper)

$$
\text { (A) }
$$

\begin{tabular}{|c|c|c|}
\hline 4207.363 & 30864 & (e) \\
\hline 4224.081 & 31117 & (e) \\
\hline 4229.812 & 30864 & (e) \\
\hline 4246.404 & 31083 & (e) \\
\hline 4252.625 & 31117 & (e) \\
\hline 4261.917 & 31169 & (e) \\
\hline 4266.180 & 31351 & (e) \\
\hline 4269.272 & 31083 & (e) \\
\hline 4275.560 & 31117 & (e) \\
\hline 4284.190 & 31083 & (e) \\
\hline 4352.600 & 31531 & (e) \\
\hline 4368.107 & 31531 & (e) \\
\hline 4507.199 & 25047 & (e) \\
\hline 4539.590 & 32603 & (e) \\
\hline 4544.694 & 25043 & (e) \\
\hline 4558.787 & 32855 & (e) \\
\hline 4565.735 & 32603 & (e) \\
\hline 4588.198 & 32837 & (e) \\
\hline 4589.895 & 32845 & (e) \\
\hline 4592.052 & 32855 & (e) \\
\hline 4616.624 & 32845 & (e) \\
\hline 4618.807 & 32855 & (e) \\
\hline 4634.073 & 32845 & (e) \\
\hline 4713.969 & 33418 & (e) \\
\hline 4737.000 & 33521 & (e) \\
\hline 4742.167 & 33418 & (e) \\
\hline 4777.778 & 30864 & (e) \\
\hline 4805.200 & 30864 & (e) \\
\hline 4824.984 & 30864 & (e) \\
\hline 4836.229 & 31117 & (e) \\
\hline 4856.190 & 31083 & (e) \\
\hline 4864.328 & 31117 & (e) \\
\hline 4876.397 & 31083 & (e) \\
\hline 4876.489 & 31169 & (e) \\
\hline 4884.603 & 31117 & (e) \\
\hline 4891.495 & 31351 & (e) \\
\hline 4920.242 & 31351 & (e) \\
\hline 4940.986 & 31351 & (e) \\
\hline 4985.413 & 31531 & (e) \\
\hline 5097.319 & 29952 & (e) \\
\hline 5116.047 & 29952 & (e) \\
\hline 5153.497 & 30307 & (e) \\
\hline 5191.434 & 30307 & (e) \\
\hline 5210.861 & 30307 & (e) \\
\hline 5246.117 & 35569 & (e) \\
\hline 5246.773 & 29952 & (e) \\
\hline 5249.435 & 30307 & (e) \\
\hline 5256.689 & 35608 & (e) \\
\hline 5267.029 & 32603 & (e) \\
\hline 5280.070 & 32855 & (e) \\
\hline 5281.065 & 35569 & (e) \\
\hline 5305.864 & 30864 & (e) \\
\hline 5308.421 & 32837 & (e) \\
\hline
\end{tabular}

$\begin{array}{ll}\text { (e) } & 2.5 \\ \text { (e) } & 2.5 \\ \text { (e) } & 2.5 \\ \text { (e) } & 1.5 \\ \text { (e) } & 2.5 \\ \text { (e) } & 3.5 \\ \text { (e) } & 2.5 \\ \text { (e) } & 1.5 \\ \text { (e) } & 2.5 \\ \text { (e) } & 1.5 \\ \text { (e) } & 1.5 \\ \text { (e) } & 1.5 \\ \text { (e) } & 2.5 \\ \text { (e) } & 2.5 \\ \text { (e) } & 1.5 \\ \text { (e) } & 2.5 \\ \text { (e) } & 2.5 \\ \text { (e) } & 3.5 \\ \text { (e) } & 1.5 \\ \text { (e) } & 2.5 \\ \text { (e) } & 1.5 \\ \text { (e) } & 2.5 \\ \text { (e) } & 1.5 \\ \text { (e) } & 2.5 \\ \text { (e) } & 3.5 \\ \text { (e) } & 2.5 \\ \text { (e) } & 2.5 \\ \text { (e) } & 2.5 \\ \text { (e) } & 2.5 \\ \text { (e) } & 2.5 \\ \text { (e) } & 1.5 \\ \text { (e) } & 2.5 \\ \text { (e) } & 1.5 \\ \text { (e) } & 3.5 \\ \text { (e) } & 2.5 \\ \text { (e) } & 2.5 \\ \text { (e) } & 2.5 \\ \text { (e) } & 2.5 \\ \text { (e) } & 1.5 \\ \text { (e) } & 0.5 \\ \text { (e) } & 0.5 \\ \text { (e) } & 1.5 \\ \text { (e) } & 1.5 \\ \text { (e) } & 1.5 \\ \text { (e) } & 2.5 \\ \text { (e) } & 0.5 \\ \text { (e) } & 1.5 \\ \text { (e) } & 3.5 \\ \text { (e) } & 2.5 \\ \text { (e) } & 2.5 \\ \text { (e) } & 2.5 \\ \text { (e) } & 2.5 \\ \text { (e) } & 3.5 \\ \text { () } & \end{array}$

$\begin{array}{ll}54626 & (\mathrm{o}) \\ 54784 & (\mathrm{o}) \\ 54499 & (\mathrm{o}) \\ 54626 & (\mathrm{o}) \\ 54626 & (\mathrm{o}) \\ 54626 & (\mathrm{o}) \\ 54784 & (\mathrm{o}) \\ 54499 & (\mathrm{o}) \\ 54499 & (\mathrm{o}) \\ 54418 & (\mathrm{o}) \\ 54499 & (\mathrm{o}) \\ 54418 & (\mathrm{o}) \\ 47227 & (\mathrm{o}) \\ 54626 & (\mathrm{o}) \\ 47040 & (\mathrm{o}) \\ 54784 & (\mathrm{o}) \\ 54499 & (\mathrm{o}) \\ 54626 & (\mathrm{o}) \\ 54626 & (\mathrm{o}) \\ 54626 & (\mathrm{o}) \\ 54499 & (\mathrm{o}) \\ 54499 & (\mathrm{o}) \\ 54418 & (\mathrm{o}) \\ 54626 & (\mathrm{o}) \\ 54626 & (\mathrm{o}) \\ 54499 & (\mathrm{o}) \\ 51789 & (\mathrm{o}) \\ 51669 & (\mathrm{o}) \\ 51584 & (\mathrm{o}) \\ 51789 & (\mathrm{o}) \\ 51669 & (\mathrm{o}) \\ 51669 & (\mathrm{o}) \\ 51584 & (\mathrm{o}) \\ 51669 & (\mathrm{o}) \\ 51584 & (\mathrm{o}) \\ 51789 & (\mathrm{o}) \\ 51669 & (\mathrm{o}) \\ 51584 & (\mathrm{o}) \\ 51584 & (\mathrm{o}) \\ 49565 & (\mathrm{o}) \\ 49493 & (\mathrm{o}) \\ 49706 & (\mathrm{o}) \\ 49565 & (\mathrm{o}) \\ 49493 & (\mathrm{o}) \\ 54626 & (\mathrm{o}) \\ 49006 & (\mathrm{o}) \\ 49352 & (\mathrm{o}) \\ 54626 & (\mathrm{o}) \\ 51584 & (\mathrm{o}) \\ 51789 & (\mathrm{o}) \\ 54499 & (\mathrm{o}) \\ 49706 & (\mathrm{o}) \\ 51669 & (\mathrm{o}) \\ & \end{array}$

2.5
3.5
1.5
2.5
2.5
2.5
3.5
1.5
1.5
0.5
1.5
0.5
3.5
2.5
2.5
3.5
1.5
2.5
2.5
2.5
1.5
1.5
0.5
2.5
2.5
1.5
3.5
2.5
1.5
3.5
2.5
2.5
1.5
2.5
1.5
3.5
2.5
1.5
1.5
1.5
0.5
2.5
1.5
0.5
2.5
1.5
2.5
2.5
1.5
3.5
1.5
2.5
2.5

$\log (g f)(\mathrm{HFR}+\mathrm{CPOL})$

$$
\begin{aligned}
& -3.99 \\
& -2.72 \\
& -2.99 \\
& -1.26 \\
& -3.45 \\
& -1.94 \\
& -1.47 \\
& -1.62 \\
& -3.66 \\
& -3.51 \\
& -3.94 \\
& -3.47 \\
& -3.98 \\
& -2.55 \\
& -2.99 \\
& -0.67 \\
& -2.45 \\
& -1.26 \\
& -1.40 \\
& -0.86 \\
& -1.05 \\
& -2.98 \\
& -2.30 \\
& -2.57 \\
& -3.97 \\
& -3.02 \\
& -3.80 \\
& -1.87 \\
& -2.00 \\
& -1.31 \\
& -1.41 \\
& -1.89 \\
& -2.05 \\
& -3.54 \\
& -3.04 \\
& -3.63 \\
& -3.67 \\
& -3.04 \\
& -3.90 \\
& -2.72 \\
& -3.52 \\
& -3.20 \\
& -3.87 \\
& -2.57 \\
& -2.64 \\
& -3.23 \\
& -3.72 \\
& -2.17 \\
& -3.48 \\
& -2.40 \\
& -1.95
\end{aligned}
$$

-2.86
-3.99
-2.72

$\log (g f)$ (Kurucz)

$\left(\mathrm{s}^{-1}\right)$

-2.475
-3.526
-3.324
-3.218

$5.29 \mathrm{E}+05$

$2.12 \mathrm{E}-05$

$7.13 \mathrm{E}+05-7.66 \mathrm{E}-04$

$3.82 \mathrm{E}+05 \quad 2.74 \mathrm{E}-04$

$6.69 \mathrm{E}+06 \quad 4.79 \mathrm{E}-03$

$2.08 \mathrm{E}+07 \quad 1.49 \mathrm{E}-02$

$\begin{array}{lll}-1.531 & 2.08 \mathrm{E}+07 & 1.49 \mathrm{E}-02 \\ -3.682 & 1.33 \mathrm{E}+05 & 7.15 \mathrm{E}-05 \\ -2.167 & 4.32 \mathrm{E}+06 & 4.64 \mathrm{E}-03\end{array}$

$\begin{array}{lll}-1.709 & 1.27 \mathrm{E}+07 & 1.37 \mathrm{E}-02 \\ -1.864 & 8.79 \mathrm{E}+06 & 1.89 \mathrm{E}-02\end{array}$

$\begin{array}{lll}-1.864 & 8.79 \mathrm{E}+06 & 1.89 \mathrm{E}-02 \\ -3.258 & 7.9 \mathrm{E}+04 & 8.48 \mathrm{E}-05\end{array}$

$7.89 \mathrm{E}+04$
$1.09 \mathrm{E}+05$

$\begin{array}{lll}-3.015 & 1.07 \mathrm{E}+05 & 2.34 \mathrm{E}-04 \\ -4.076 & 3.75 \mathrm{E}+04 & 1.92 \mathrm{E}-05\end{array}$

$\begin{array}{lll}-2.530 & 1.10 \mathrm{E}+05 & 7.88 \mathrm{E}-05\end{array}$

$\begin{array}{lll}-2.530 & -1.10 \mathrm{E}+05 & 7.88 \mathrm{E}-05 \\ -4.022 & 3.37 \mathrm{E}+04 & 2.36 \mathrm{E}-05\end{array}$

$\begin{array}{lll}-4.022 & 3.862 & \\ -2.462 & 3.78 \mathrm{E}+05 & 04\end{array}$

$\begin{array}{lll}-2.462 & 8.89 \mathrm{E}+05 & 4.78 \mathrm{E}-04 \\ -2.110 & 3.31 \mathrm{E}+05 & 3.56 \mathrm{E}-04\end{array}$

$\begin{array}{lll}-2.110 & 3.31 \mathrm{E}+05 & 3.56 \mathrm{E}-04 \\ -0.630 & 6.55 \mathrm{E}+07 & 4.69 \mathrm{E}-02\end{array}$

$\begin{array}{lll}-0.630 & 6.55 \mathrm{E}+07 & 4.69 \mathrm{E}-02 \\ -2.660 & 1.08 \mathrm{E}+06 & 7.74 \mathrm{E}-04\end{array}$

$\begin{array}{lll}-2.660 & 1.08 \mathrm{E}+06 & 7.74 \mathrm{E}-04 \\ -1.220 & 1.69 \mathrm{E}+07 & 1.21 \mathrm{E}-02\end{array}$

$\begin{array}{lll}-1.220 & 1.69 \mathrm{E}+07 & 1.21 \mathrm{E}-02 \\ -1.290 & 1.22 \mathrm{E}+07 & 1.31 \mathrm{E}-02\end{array}$

$\begin{array}{lll}-1.290 & 1.22 \mathrm{E}+07 & 1.31 \mathrm{E}-02 \\ -1.110 & 4.17 \mathrm{E}+07 & 4.48 \mathrm{E}-02\end{array}$

$\begin{array}{rrr}-1.110 & 4.17 \mathrm{E}+07 & 4.48 \mathrm{E}-02 \\ -1240 & 2.68 \mathrm{E}+07 & 5.76 \mathrm{E}-02\end{array}$

$\begin{array}{lll}-1.240 & 2.68 \mathrm{E}+07 & 5.76 \mathrm{E}-02 \\ -3.559 & 3.09 \mathrm{E}+05 & 2.21 \mathrm{E}-04\end{array}$

$\begin{array}{lll}-2.782 & 1.48 \mathrm{E}+06 & 1.06 \mathrm{E}-03\end{array}$

$\begin{array}{lll}-3.125 & 7.94 \mathrm{E}+05 & 8.54 \mathrm{E}-04\end{array}$

$\begin{array}{lll}-4.758 & 3.11 \mathrm{E}+04 & 1.59 \mathrm{E}-05 \\ - & 2.583 & 1.89 \mathrm{E}-04\end{array}$

$\begin{array}{lll}-4.758 & 3.11 \mathrm{E}+04 & 1.59 \mathrm{E}-05 \\ -4.583 & 2.76 \mathrm{E}+05 & 1.89 \mathrm{E}-04\end{array}$

$\begin{array}{lll} & 4.51 \mathrm{E}+04 & 4.74 \mathrm{E}-05 \\ -2.250 & 3.87 \mathrm{E}+06 & 1.98 \mathrm{E}-03\end{array}$

$\begin{array}{lll}-2.250 & 3.87 \mathrm{E}+06 & 1.98 \mathrm{E}-03 \\ -2.260 & 2.84 \mathrm{E}+06 & 1.94 \mathrm{E}-03\end{array}$

$\begin{array}{lll}-2.260 & 2.84 \mathrm{E}+06 & 1.94 \mathrm{E}-03 \\ -1.372 & 1.40 \mathrm{E}+07 & 9.57 \mathrm{E}-03\end{array}$

$\begin{array}{lll}-1.372 & 1.40 \mathrm{E}+07 & 9.57 \mathrm{E}-03 \\ -1.457 & 1.09 \mathrm{E}+07 & 1.14 \mathrm{E}-02\end{array}$

$\begin{array}{lll}-1.460 & 3.62 \mathrm{E}+06 & 1.14 \mathrm{E}-02 \\ -2.080 & 2.47 \mathrm{E}-03\end{array}$

$\begin{array}{lll}-2.080 & 2.53 \mathrm{E}+06 & 2.66 \mathrm{E}-03 \\ -3.044 & 8.10 \mathrm{E}+04 & 4.15 \mathrm{E}-05\end{array}$

$\begin{array}{lll}-3.044 & 8.10 \mathrm{E}+04 & 4.15 \mathrm{E}-05 \\ -2.488 & 2.52 \mathrm{E}+05 & 1.72 \mathrm{E}-04\end{array}$

-3.091
-3.932

-3.932
-2.640

$-3.630$

-2.696
-3.362

-3.362
-2.945

-2.945
-4.167

$-2.450$

$-2.426$

-3.640
-3.324

$-2.011$

$-3.863$

-2.357
-1.810

$2.52 \mathrm{E}+05$

$6.41 \mathrm{E}+04$
$5.67 \mathrm{E}+04$

$1.72 \mathrm{E}-04$

$6.73 \mathrm{E}-05$

$5.95 \mathrm{E}-05$

$2.47 \mathrm{E}-04$

$7.01 \mathrm{E}-05$

$3.61 \mathrm{E}-04$

$7.91 \mathrm{E}-05$

$3.35 \mathrm{E}-04$

$2.42 \mathrm{E}-05$

$7.78 \mathrm{E}-04$

$1.04 \mathrm{E}-04$

$4.79 \mathrm{E}-05$

$7.94 \mathrm{E}-04$

$7.94 \mathrm{E}-04$
$8.63 \mathrm{E}-05$

$\begin{array}{ll}8.03 \mathrm{E}+04 & 8.63 \mathrm{E}-05 \\ 9.59 \mathrm{E}+05 & 7.19 \mathrm{E}-04 \\ 2.52 \mathrm{E}+06 & 1.72 \mathrm{E}-03\end{array}$

$\begin{array}{ll}8.03 \mathrm{E}+04 & 8.63 \mathrm{E}-05 \\ 9.59 \mathrm{E}+05 & 7.19 \mathrm{E}-04 \\ 2.52 \mathrm{E}+06 & 1.72 \mathrm{E}-03\end{array}$

(continued on next page) 
Table 6 (continued)

\begin{tabular}{|c|c|c|c|c|c|c|c|c|c|c|}
\hline $\begin{array}{l}\text { Wavelength } \\
(\AA)\end{array}$ & $\begin{array}{l}E \text { (Lower) } \\
\left(\mathrm{cm}^{-1}\right)\end{array}$ & Parity (Lower) & $J$ (Lower) & $\begin{array}{l}E \text { (Upper) } \\
\left(\mathrm{cm}^{-1}\right)\end{array}$ & Parity (Upper) & $J$ (Upper) & $\log (g f)(\mathrm{HFR}+\mathrm{CPOL})$ & $\log (g f)($ Kurucz) & $\begin{array}{l}g A(\mathrm{HFR}+\mathrm{CPOL}) \\
\left(\mathrm{s}^{-1}\right)\end{array}$ & $B F(\mathrm{HFR}+\mathrm{CPOL})$ \\
\hline 5310.692 & 32845 & (e) & 1.5 & 51669 & (o) & 2.5 & -2.29 & -2.280 & $1.15 \mathrm{E}+06$ & $7.86 \mathrm{E}-04$ \\
\hline 5313.581 & 32855 & (e) & 2.5 & 51669 & (o) & 2.5 & -1.63 & -1.650 & $5.31 \mathrm{E}+06$ & $3.63 \mathrm{E}-03$ \\
\hline 5318.387 & 29952 & (e) & 0.5 & 48749 & (o) & 0.5 & -3.28 & -3.128 & $1.26 \mathrm{E}+05$ & $3.15 \mathrm{E}-04$ \\
\hline 5334.868 & 32845 & (e) & 1.5 & 51584 & (o) & 1.5 & -1.70 & -1.562 & $4.40 \mathrm{E}+06$ & $4.62 \mathrm{E}-03$ \\
\hline 5337.783 & 32855 & (e) & 2.5 & 51584 & (o) & 1.5 & -2.12 & -2.029 & $1.69 \mathrm{E}+06$ & $1.77 \mathrm{E}-03$ \\
\hline 5346.086 & 30864 & (e) & 2.5 & 49565 & (o) & 1.5 & -3.09 & -2.650 & $1.93 \mathrm{E}+05$ & $2.03 \mathrm{E}-04$ \\
\hline 5346.541 & 30307 & (e) & 1.5 & 49006 & (o) & 1.5 & -3.09 & -2.948 & $1.92 \mathrm{E}+05$ & $2.26 \mathrm{E}-04$ \\
\hline 5378.048 & 31117 & (e) & 2.5 & 49706 & (o) & 2.5 & -3.68 & -4.400 & $4.90 \mathrm{E}+04$ & $3.68 \mathrm{E}-05$ \\
\hline 5396.881 & 36102 & (e) & 3.5 & 54626 & (o) & 2.5 & -3.60 & -3.592 & $6.03 \mathrm{E}+04$ & 4.32E-05 \\
\hline 5407.615 & 30864 & (e) & 2.5 & 49352 & (o) & 2.5 & -2.33 & -2.088 & $1.08 \mathrm{E}+06$ & $8.28 \mathrm{E}-04$ \\
\hline 5420.925 & 30307 & (e) & 1.5 & 48749 & (o) & 0.5 & -2.61 & -2.360 & $5.71 \mathrm{E}+05$ & $1.43 \mathrm{E}-03$ \\
\hline 5441.898 & 33418 & (e) & 2.5 & 51789 & (o) & 3.5 & -3.88 & -4.260 & $2.90 \mathrm{E}+04$ & $1.49 \mathrm{E}-05$ \\
\hline 5477.502 & 33418 & (e) & 2.5 & 51669 & (o) & 2.5 & -2.89 & -2.994 & $2.80 \mathrm{E}+05$ & $1.91 \mathrm{E}-04$ \\
\hline 5503.224 & 33418 & (e) & 2.5 & 51584 & (o) & 1.5 & -2.60 & -2.306 & $5.33 \mathrm{E}+05$ & $5.60 \mathrm{E}-04$ \\
\hline 5508.623 & 33521 & (e) & 3.5 & 51669 & (o) & 2.5 & -2.48 & -2.110 & $6.96 \mathrm{E}+05$ & $4.76 \mathrm{E}-04$ \\
\hline 5510.718 & 30864 & (e) & 2.5 & 49006 & (o) & 1.5 & -2.62 & -2.452 & $5.30 \mathrm{E}+05$ & $6.23 \mathrm{E}-04$ \\
\hline 5926.178 & 32837 & (e) & 3.5 & 49706 & (o) & 2.5 & -4.00 & -4.354 & $1.85 \mathrm{E}+04$ & $1.39 \mathrm{E}-05$ \\
\hline 6070.107 & 38315 & (e) & 2.5 & 54784 & (o) & 3.5 & -3.19 & -2.944 & $1.10 \mathrm{E}+05$ & $5.91 \mathrm{E}-05$ \\
\hline 6112.263 & 38270 & (e) & 3.5 & 54626 & (o) & 2.5 & -3.14 & -2.943 & $1.22 \mathrm{E}+05$ & $8.74 \mathrm{E}-05$ \\
\hline 6129.226 & 38315 & (e) & 2.5 & 54626 & (o) & 2.5 & -2.71 & -2.440 & $3.29 \mathrm{E}+05$ & $2.36 \mathrm{E}-04$ \\
\hline 6147.146 & 38362 & (e) & 1.5 & 54626 & (o) & 2.5 & -3.08 & -2.843 & $1.38 \mathrm{E}+05$ & $9.89 \mathrm{E}-05$ \\
\hline 6176.982 & 38315 & (e) & 2.5 & 54499 & (o) & 1.5 & -3.05 & -2.841 & $1.49 \mathrm{E}+05$ & $1.60 \mathrm{E}-04$ \\
\hline 6195.183 & 38362 & (e) & 1.5 & 54499 & (o) & 1.5 & -3.04 & -2.802 & $1.52 \mathrm{E}+05$ & $1.63 \mathrm{E}-04$ \\
\hline 6208.189 & 38396 & (e) & 0.5 & 54499 & (o) & 1.5 & -3.22 & -2.984 & $9.97 \mathrm{E}+04$ & $1.07 \mathrm{E}-04$ \\
\hline 6209.376 & 35569 & (e) & 2.5 & 51669 & (o) & 2.5 & -3.99 & -4.233 & $1.78 \mathrm{E}+04$ & $1.22 \mathrm{E}-05$ \\
\hline 6226.645 & 38362 & (e) & 1.5 & 54418 & (o) & 0.5 & -3.19 & -2.981 & $1.04 \mathrm{E}+05$ & $2.24 \mathrm{E}-04$ \\
\hline 6239.783 & 38396 & (e) & 0.5 & 54418 & (o) & 0.5 & -3.22 & -2.991 & $9.79 \mathrm{E}+04$ & $2.10 \mathrm{E}-04$ \\
\hline 7419.668 & 38315 & (e) & 2.5 & 51789 & (o) & 3.5 & -2.55 & -2.424 & $3.15 \mathrm{E}+05$ & $1.61 \mathrm{E}-04$ \\
\hline 7486.011 & 38315 & (e) & 2.5 & 51669 & (o) & 2.5 & -3.15 & -3.014 & $7.79 E+04$ & $5.32 \mathrm{E}-05$ \\
\hline 7512.760 & 38362 & (e) & 1.5 & 51669 & (o) & 2.5 & -2.76 & -2.630 & $1.90 \mathrm{E}+05$ & $1.30 \mathrm{E}-04$ \\
\hline 7561.233 & 38362 & (e) & 1.5 & 51584 & (o) & 1.5 & -3.30 & -3.172 & $5.32 \mathrm{E}+04$ & $5.59 \mathrm{E}-05$ \\
\hline 7580.615 & 38396 & (e) & 0.5 & 51584 & (o) & 1.5 & -2.97 & -2.838 & $1.15 \mathrm{E}+05$ & $1.21 \mathrm{E}-04$ \\
\hline 18072.536 & 49352 & (o) & 2.5 & 54883 & (e) & 2.5 & -3.92 & & $2.38 \mathrm{E}+03$ & \\
\hline 52704.748 & 51669 & (o) & 2.5 & 53566 & (e) & 3.5 & -3.99 & -3.869 & $3.02 \mathrm{E}+02$ & \\
\hline 62420.503 & 51669 & (o) & 2.5 & 53271 & (e) & 2.5 & -3.58 & -3.492 & $5.75 \mathrm{E}+02$ & \\
\hline 68136.875 & 51584 & (o) & 1.5 & 53051 & (e) & 1.5 & -3.70 & -3.628 & $3.75 \mathrm{E}+02$ & \\
\hline 69034.463 & 53051 & (e) & 1.5 & 54499 & (o) & 1.5 & -3.80 & -3.673 & $2.03 \mathrm{E}+02$ & $2.18 \mathrm{E}-07$ \\
\hline 73153.230 & 53051 & (e) & 1.5 & 54418 & (o) & 0.5 & -3.46 & -3.325 & $3.95 \mathrm{E}+02$ & $8.49 \mathrm{E}-07$ \\
\hline 73803.061 & 53271 & (e) & 2.5 & 54626 & (o) & 2.5 & -3.71 & -3.577 & $2.22 \mathrm{E}+02$ & $1.59 \mathrm{E}-07$ \\
\hline 81378.953 & 53271 & (e) & 2.5 & 54499 & (o) & 1.5 & -3.31 & -3.174 & $4.46 \mathrm{E}+02$ & $4.79 \mathrm{E}-07$ \\
\hline 94372.311 & 53566 & (e) & 3.5 & 54626 & (o) & 2.5 & -3.20 & -3.057 & $4.32 \mathrm{E}+02$ & $3.10 \mathrm{E}-07$ \\
\hline
\end{tabular}

